\title{
STOCHASTIC STOKES' DRIFT, HOMOGENIZED FUNCTIONAL INEQUALITIES, AND LARGE TIME BEHAVIOR OF BROWNIAN RATCHETS
}

\author{
ADRIEN BLANCHET*, JEAN DOLBEAULT ${ }^{\dagger}$, AND MICHAł KOWALCZYK ${ }^{\ddagger}$
}

\begin{abstract}
A periodic perturbation of a Gaussian measure modifies the sharp constants in Poincaré and logarithmic Sobolev inequalities in the homogenization limit, that is, when the period of a periodic perturbation converges to zero. We use variational techniques to determine the homogenized constants and get optimal convergence rates towards equilibrium of the solutions of the perturbed diffusion equations.

The study of these sharp constants is motivated by the study of the stochastic Stokes' drift. It also applies to Brownian ratchets and molecular motors in biology. We first establish a transport phenomenon. Asymptotically, the center of mass of the solution moves with a constant velocity, which is determined by a doubly periodic problem. In the reference frame attached to the center of mass, the behavior of the solution is governed at large scale by a diffusion with a modified diffusion coefficient. Using the homogenized logarithmic Sobolev inequality, we prove that the solution converges in selfsimilar variables attached to the center of mass to a stationary solution of a Fokker-Planck equation modulated by a periodic perturbation with fast oscillations, with an explicit rate. We also give an asymptotic expansion of the traveling diffusion front corresponding to the stochastic Stokes' drift with given potential flow.

Key words. Stochastic Stokes' drift; Brownian ratchets; molecular motors; asymptotic expansion; doubly-periodic equation; Fokker-Planck equation; moment estimates; contraction; transport; traveling potential; traveling front; effective diffusion; intermediate asymptotics; functional inequalities; sharp constants; Poincaré inequality; spectral gap; generalized Poincaré inequalities; spectral gap; Holley-Stroock perturbation results; logarithmic Sobolev inequalities; interpolation; perturbation; homogenization; two-scale convergence; minimizing sequences; defect of convergence; loss of compactness.
\end{abstract}

MSC (2000): Primary: 26D15, 35B27; Secondary: 60E15, 80M40

1. Introduction. This paper is devoted to the analysis of the large time behavior of the solution of

$$
f_{t}=\Delta f+\nabla \cdot[\nabla \psi(x-\omega t \mathrm{e}) f], \quad x \in \mathbb{R}^{d}, t>0 .
$$

We are interested in the case where $\psi$ is continuous, periodic: $\psi(y+\mathbf{k})=\psi(y)$ for any $(y, \mathbf{k}) \in \mathbb{R}^{d} \times \mathbb{Z}^{d}$, and will simply write $\psi$ as a function of $y \in \mathbb{T}^{d} \approx[0,1)^{d}$. Furthermore $\omega \in \mathbb{R}$ is a constant and $\mathrm{e} \in \mathbb{R}^{d}$ is a fixed vector, such that $|\mathrm{e}|=1$. With these notations, $\psi(x-\omega t \mathrm{e})$ represents a periodic potential in $\mathbb{R}^{d}$ moving with a constant speed $\omega$ in the direction of the vector e, that is a traveling potential.

Problem (1.1) is a simple model describing diffusion of particles in the presence of a periodic, wave-like potential. This problem is known as the stochastic Stokes' drift, see [11], a model in which particles suspended in a liquid and subject to diffusion experience a net drift due to a wave traveling through the liquid. When $\psi$ is periodic but asymmetric, (1.1) is also a simple model of Brownian ratchet. When there is no diffusion, the net drift of particles is equal to $\omega$ when $\omega$ is small, but decays to 0 when $\omega$ is large. One may expect that in the presence of a diffusion the situation is different since, due to the Brownian motion, some particles will move in the direction

* Department of Applied Mathematics and Theoretical Physics, Centre for Mathematical Sciences, Wilberforce Road, Cambridge CB3 0WA, United Kingdom. E-mail: adrien.blanchet@univ-tlse1.fr

†Ceremade (UMR CNRS no. 7534), Université Paris-Dauphine, Place de Lattre de Tassigny, 75775 Paris Cédex 16, France. E-mail: dolbeaul@ceremade.dauphine.fr

$\ddagger$ Universidad de Chile, Facultad de Ciencias Físicas y Matemáticas, Depto Ingeniería Matemática, Blanco Encalada 2120, Piso 5, Santiago, Chile. E-mail: kowalczy@dim.uchile.cl 
opposite to the wave train. This is indeed the case and the asymptotic speed of the center of mass is decreased by the diffusion. The effective diffusion of the particles is also changed by the traveling wave. Surprisingly, it can be decreased or increased, depending on $\omega$, an effect which is apparently not mentioned in the physics literature. This last statement is perhaps less obvious although similar effects are already known in the context of homogenization theory, see e.g., $[26,45]$. To address the mutual influence of transport and diffusion in the stochastic Stokes' drift, we will analyze the large time asymptotic profiles of solutions of (1.1). A first step will be to characterize the speed of the traveling front and to show that it is asymptotically the same as the speed of the center of mass of the solution. Then, in the reference frame attached to the center of mass, a time rescaling transforms the traveling potential into an oscillating term whose influence on the large time behavior can be understood using the tools of homogenization theory. Moreover, several length scales have to be taken into account. The position of the center of mass is of the order of $t$, while the typical size of the front grows like $\sqrt{t}$. Typical relaxation rates are exponential at small scale, but of the order of $1 / \sqrt{t}$ when measured globally in $L^{1}$.

A key tool for the understanding of the stochastic Stokes' drift rewritten in selfsimilar variables attached to the center of mass, is the logarithmic Sobolev inequality for a Gaussian measure perturbed by a bounded oscillating potential, namely $d \mu_{\varepsilon}(x):=Z_{\varepsilon}^{-1} e^{-\phi(x / \varepsilon)-|x|^{2} / 2} d x$. Most of this paper is devoted to the analysis of the large time behavior of the solution of

$$
u_{t}^{\varepsilon}=\Delta u^{\varepsilon}+\nabla \cdot\left[x u^{\varepsilon}+\frac{1}{\varepsilon} \nabla \phi\left(\frac{x}{\varepsilon}\right) u^{\varepsilon}\right], \quad x \in \mathbb{R}^{d}, t>0,
$$

for $\varepsilon \rightarrow 0_{+}$. As for $\psi$, we shall assume that $\phi$ is of class $C^{2}$, periodic: $\phi(y+k)=\phi(y)$ for any $(y, k) \in \mathbb{R}^{d} \times \mathbb{Z}^{d}$, and simply write $\phi$ as a function of $y \in \mathbb{T}^{d} \approx[0,1)^{d}$. If $\omega=0$, one can expect that (1.2) with $\phi=\psi$ gives a description of the large time behavior of the solution of (1.1) in self-similar variables, with $\varepsilon \sim 1 / \sqrt{t}$ as $t \rightarrow \infty$. In the analysis of (1.1), $\phi$ and $\psi$ are related but not equal if $\omega \neq 0$.

Problem (1.2) has already been studied in the context of diffusive turbulent flows, see $[25,36]$ and references therein. In particular in [25], and in a much more general framework than ours, the homogenized limit of (1.2) is considered and the results are stated in terms of bounded measures. Here we focus on the case of one-dimensional potential flows. By using logarithmic Sobolev inequalities, we give a different type of results based on the relative entropy with respect to the solution found by a formal asymptotic expansion. Using the associated logarithmic Sobolev inequalities, we obtain stronger convergence results for the solutions of (1.1): the two-scale convergence of measures is replaced by a strong convergence in $L^{1}$, and estimates of the rates are deduced from the sharp constants in logarithmic Sobolev inequalities.

The literature on the stochastic Stokes' drift and Brownian ratchets is huge. Let us mention a few introductory papers, and contributions which are relevant for our purpose, from the physics point of view. As for the stochastic Stokes' drift, we first refer to [30], which contains many results of interest for our paper: there the asymptotic speed of the center of mass, or drift velocity, is computed in the case of a sinusoidal traveling potential (also see $[35,21]$ ) and the diffuse traveling front is exhibited on the basis of numerical results.

Brownian ratchets generically refer to drift-diffusion models in which a time periodic forcing coupled to some asymmetry induces a transport at large scale which 
would not occur without an explicit time-dependence. The denomination of Brownian ratchets covers a large variety of models, which are believed to be of fundamental importance for the description of motion at sub-cellular scale in mathematical biology. Rocking ratchet models are related with traveling potentials, see [11]. The notion of traveling potential and the connection with Brownian ratchets is explored in [15, 27]. We refer to [40] for the notion of tilted Smoluchowski-Feynman ratchet, which makes an explicit connection between the stochastic Stokes' drift and ratchet mechanisms. See Remark 2.1 for more details. For introductory papers to ratchets and their applications in biology, see [35, 12, 41, 1, 7, 31,33]. For more insight from the physics point of view, see [40]. A broad historical perspective is given in [27]. An attempt of typology can be found for instance in [41]. We will not give specific references for instance to the ratchet and pawl model considered by M. v. Smoluchowski and R. Feynman and suggest the interested reader to refer to one of the papers quoted above. There are also many applications of ratchet models, for instance to SQUID devices, which are out of the scope of this paper. Some issues, like effects due to the asymmetry of the potential, which are important in specific contexts, are not relevant for our approach, but corresponding references can be found in the above review papers as well.

As far as mathematical issues are concerned, one can quote $[32,19,18,24,9$, $28,38]$. We also refer to the recently published book of B. Perthame, [37], for a broader overview of transport issues in mathematical biology. Other references concerning mathematical methods which are not directly connected with the stochastic Stokes' drift or Brownian ratchets, like results on functional inequalities or methods of homogenization theory, will be quoted when needed in the paper.

This paper is focused on the mathematical description of the intermediate asymptotics of (1.1) and on the inequalities which govern the behavior of the solutions of (1.2). Qualitative properties - some of them are mentioned without proof in this paper - are more relevant from the physics point of view and have been described elsewhere, see [14]. Here we first study the large time behavior of the solutions of the stochastic Stokes' drift. We perform a formal asymptotic expansion and give a sketch of a proof in Section 2. The key tool is the logarithmic Sobolev inequality for the measure $d \mu_{\varepsilon}$, which corresponds to the unique stationary Gibbs state of (1.2). The main effort in this paper is directed towards the study of the homogenized limit of a family of functional inequalities, which interpolate between Poincaré and logarithmic Sobolev inequalities, and govern the rate of convergence to equilibrium for (1.2), see Sections 3 and 4. More precisely, we are interested in the limit of the sharp constants of these inequalities as $\varepsilon \rightarrow 0_{+}$. We give a formal expansion in Section 3, state the main result in Theorem 4.1, and give its proof in Sections 5 and 6 using a variational approach. A statement on rates of convergence for the solutions of (1.2) is given in Section 7 .

2. Stochastic Stokes' drift and logarithmic Sobolev inequalities. Consider a solution of (1.1). A first case, which is particularly simple, is the case $\omega=0$. Let $R(t):=\sqrt{1+2 t}$. The function $u$ defined by the change of coordinates

$$
f(t, x)=\frac{1}{R^{d}(t)} u\left(\log R(t), \frac{x}{R(t)}\right)
$$


is a solution of

$$
\begin{cases}u_{t}=\Delta u+\nabla \cdot(x u)+R \nabla \cdot(u \nabla \psi(R x)), & x \in \mathbb{R}^{d}, t>0, \\ u(t=0, x)=f_{0}(x), & x \in \mathbb{R}^{d},\end{cases}
$$

where, in the new variables,

$$
R(t)=e^{t} \quad \forall t>0 .
$$

For large values of $t$, we can formally regard $\varepsilon=1 / R(t)$ as a small parameter and it is reasonable to expect that the behavior of the solution is well described by (1.2) with $\phi=\psi$ in the limit $\varepsilon \rightarrow 0_{+}$.

When $\omega>0$, Equation (1.2) is also going to play a role in the large time behavior of the solutions of (1.1), but the description is not as simple as above. The combination of the drift, which is time-periodic, and of the diffusion induces a motion of the center of mass. The speed of displacement is known as the ballistic velocity, see [26, 45], or drift velocity, see [35, 21]. This velocity can be characterized with the help of the periodic problem in $\mathbb{T}^{d} \subset \mathbb{R}^{d}$

$$
\begin{cases}g_{t}=\Delta g+\nabla \cdot(g \nabla \psi(x-\omega t \mathrm{e})), & x \in \mathbb{T}^{d}, t>0, \\ g(t=0, x)=g_{0}(x)=\sum_{k \in \mathbb{Z}} f_{0}(x+k), & x \in \mathbb{T}^{d},\end{cases}
$$

for which, by linearity of the equations (see [40] for more details), we get

$$
g(t, x)=\sum_{k \in \mathbb{Z}^{d}} f(t, x+k) \quad \forall(t, x) \in \mathbb{R}^{+} \times \mathbb{T}^{d} .
$$

Like in [24], one can prove that $g$ converges exponentially fast to a time-periodic solution $g_{\infty}$ of (2.1) under some technical assumptions on $\psi$. The solution is unique by a contraction property along the flow, and so is also the unique time-periodic solution. Now, let us make the link with the position of the center of mass of the solution of (1.1).

Consider now a solution $f$ of (1.1). Assume for simplicity that $\int_{\mathbb{R}^{d}} f_{0} d x=1$. Then $\int_{\mathbb{R}^{d}} f(t, \cdot) d x=1$ for any $t \geq 0$ and we can define the position of the center of mass by

$$
\bar{x}(t):=\int_{\mathbb{R}^{d}} x f(t, x) d x .
$$

An integration by parts shows that

$$
\begin{aligned}
\frac{d \bar{x}}{d t}=\int_{\mathbb{R}^{d}} x f_{t} d x & =-d \int_{\mathbb{R}^{d}} \nabla \psi(x-\omega t \mathrm{e}) f(t, x) d x \\
& =-d \sum_{k \in \mathbb{Z}^{d}} \int_{\mathbb{T}^{d}} \nabla \psi(x-\omega t \mathrm{e}) f(t, x+k) d x \\
& =-d \int_{\mathbb{T}^{d}} \nabla \psi(x-\omega t \mathrm{e}) g(t, x) d x \\
& \underset{t \rightarrow \infty}{\sim}-d \int_{\mathbb{T}^{d}} \nabla \psi(x-\omega t \mathrm{e}) g_{\infty}(t, x) d x .
\end{aligned}
$$


If we define

$$
c=-d \int_{0}^{1} d t \int_{\mathbb{T}^{d}} \nabla \psi(x-\omega t \mathrm{e}) g_{\infty}(t, x) d x
$$

then a more careful analysis of (2.1) shows that $\frac{d \bar{x}}{d t}-c$ converges to 0 at an exponential rate. Hence

$$
\bar{x}(t) \sim c t \quad \text { as } t \rightarrow \infty
$$

and it makes sense to introduce the change of coordinates

$$
f(t, x)=\frac{1}{R^{d}} u\left(\log R, \frac{x-c t \mathrm{e}}{R}\right),
$$

with $R(t)=\sqrt{1+2 t}$ as above, in order to understand the large time behavior of $f$. In the new variables, the equation is

$$
u_{t}=\Delta u+\nabla(x u)+R \nabla \cdot\left[u\left(c \mathrm{e}+\nabla \psi\left(R x+\frac{1}{2}\left(R^{2}-1\right)(c-\omega) \mathrm{e}\right)\right)\right] .
$$

At this point, we shall assume that $d=1$ to simplify the discussion. The higher dimensional case is similar. The time-periodic solution $g_{\infty}$ can also be written as a function of $x-\omega t$ (here $\mathrm{e}=1$ ) since the solution is unique and can be obtained as follows. The function $g_{\infty}(t, x)=g_{\omega}(x-\omega t)$ solves the equation

$$
\left(g_{\omega}\right)_{x x}+\left(\left(\omega+\psi^{\prime}\right) g_{\omega}\right)_{x}=0,
$$

with periodic boundary conditions. If we take a primitive of $(2.4)$, we get that

$$
x \mapsto\left(g_{\omega}\right)_{x}+\left(\omega+\psi^{\prime}\right) g_{\omega}=: A(\omega)
$$

is constant. By taking one more integral of (2.5), using the normalization condition $\int_{0}^{1} g_{\omega}(x) d x=1$ and the definition of $c=c(\omega)$ given by $(2.2)$, we get that

$$
\omega-c(\omega)=\omega \int_{0}^{1} g_{\omega} d x+\int_{0}^{1} \psi^{\prime} g_{\omega} d x=A(\omega) .
$$

Some elementary but tedious computations show that $c(\omega)<\omega, \lim _{\omega \rightarrow 0_{+}} c(\omega) / \omega>0$, $c(\omega)$ is positive for large values of $\omega$, and $\lim _{\omega \rightarrow \infty} c(\omega)=0$, see [14].

REMARK 2.1. We observe that in general we have $|c(\omega)| \neq|c(-\omega)|$, when $\psi$ has no simple symmetry, which means that the average speed of particles in the stochastic Stokes' drift depends on the direction in which the potential moves. This feature of (1.1) is reminiscent of the Brownian ratchet mechanism. Actually, if $f$ is a solution of (1.1), we may observe that $\tilde{f}(t, x)=f(t, x-\omega t)$ is a solution of

$$
\tilde{f}_{t}=\tilde{f}_{x x}+\left(\left(\omega+\psi^{\prime}\right) \tilde{f}\right)_{x}, \quad x \in \mathbb{R}, t>0,
$$

a problem which is known as the tilted Smoluchowski-Feynman ratchet, see for instance [40]. For more general drifts, this equation has also been considered from a physics point of view for the understanding of flow reversals, under the condition that $\psi$ is explicitly time-dependent, see for instance [7, 13, 22, 39, 41]. 
We continue the analysis of the stochastic Stokes' drift. After rescaling, the equation for $u$ is

$$
u_{t}=u_{x x}+(x u)_{x}+R\left[\left(\psi^{\prime}\left(R x-\frac{1}{2}\left(R^{2}-1\right) A(\omega)\right)+c(\omega)\right) u\right]_{x}
$$

with $R(t)=e^{t}$. If we had $\omega=c(\omega)$, we would get $A(\omega)=0$ and the analysis would then be the same as in the case $\omega=0$. Such an equality is however false, and a more detailed analysis is required. Let us continue our heuristic approach by introducing a two-scale function $\mathrm{U}$. Since $f$ in (2.3) only depends on $x-c t$ e, only one variable, $z$, is needed at small scale. Let

$$
u(t, x)=\mathrm{U}(t, x ; z) \quad \text { with } \quad z:=R x-\frac{1}{2}\left(R^{2}-1\right) A(\omega),
$$

in the large $R=e^{t}$ limit. Using the chain rule, we see that $U$ should be solution of

$\mathrm{U}_{t}=R^{2}\left(\mathrm{U}_{z z}+A \mathrm{U}_{z}+\left[\left(\psi^{\prime}(z)+c\right) \mathrm{U}\right]_{z}\right)+R\left(2 \mathrm{U}_{z}+\left(\psi^{\prime}(z)+c\right) \mathrm{U}_{x}+\left(\mathrm{U}_{x x}+(x \mathrm{U})_{x}\right)\right.$.

Using $c(\omega)+A(\omega)=\omega$, we can rewrite the equation for $\mathrm{U}$ as

$$
\mathrm{U}_{t}-\mathrm{U}_{x x}-(x \mathrm{U})_{x}=R^{2}\left(\mathrm{U}_{z z}+\left(\left(\omega+\psi^{\prime}(z)\right) \mathrm{U}\right)_{z}\right)+R\left(2 \mathrm{U}_{z}+\left(\psi^{\prime}(z)+c\right) \mathrm{U}\right)_{x}
$$

where $c=c(\omega)$. We will formally solve the equation order by order.

(i) In order to cancel the terms of order $R^{2}$ in the equation, $U$ has to be proportional to $g_{\omega}$ because of (2.4). Therefore, let us introduce the ansatz

$$
\mathrm{U}(t, x ; z)=g_{\omega}(z) h(t, x)+R^{-1} \mathrm{U}^{(1)}(t, x ; z)+O\left(R^{-2}\right) .
$$

(ii) At order $R$, we find that

$$
\mathrm{U}^{(1)}(t, x ; z)=g_{\omega}^{(1)}(z) h_{x}(t, x)
$$

where $g_{\omega}^{(1)}$ is given as a solution of the equation

$$
\left(g_{\omega}^{(1)}\right)_{z z}+\left(\left(\omega+\psi^{\prime}(z)\right) g_{\omega}^{(1)}\right)_{z}=-2\left(g_{\omega}\right)_{z}-\left(\psi^{\prime}(z)+c\right) g_{\omega} .
$$

There is a solvability condition at order $R$ : the average on $(0,1)$ of the right hand side of the equation is 0 . Since all functions are periodic and $\int_{0}^{1} g_{\omega}(z) d z=1$, we recover the condition

$$
\int_{0}^{1} \psi^{\prime}(z) g_{\omega}(z) d z+c(\omega)=0
$$

which has already been found above by consideration on the position of the center of mass. Reciprocally, if the solvability condition is fulfilled, (2.8) has a solution. Notice that $g_{\omega}^{(1)}$ is unique up to the addition of a constant and a multiple of $g_{\omega}$. Further assume that

$$
\int_{0}^{1} g_{\omega}^{(1)}(z) d z=0
$$

(iii) At order $R^{0}=1$, the solvability condition is:

$$
h_{t}-h_{x x}-(x h)_{x}=h_{x x} \int_{6}^{1}\left(\psi^{\prime}(z)+c\right) g_{\omega}^{(1)}(z) d z .
$$


Hence we obtain a modified Fokker-Planck equation

$$
h_{t}=\kappa_{\omega} h_{x x}+(x h)_{x}
$$

where, using (2.9), the effective diffusion coefficient is given by

$$
\kappa_{\omega}:=1+\int_{0}^{1} \psi^{\prime}(z) g_{\omega}^{(1)}(z) d z
$$

Summarizing we formally have that

$$
\mathrm{U}(t, x ; z)=g_{\omega}(z) h(t, x)+g_{\omega}^{(1)}(z) h_{x}(t, x)+O\left(R^{-2}\right),
$$

where $g_{\omega}, g_{\omega}^{(1)}$ and $h$ are respectively defined in (2.4), (2.8) and (2.10). As we are interested in the large time behavior, we can use a more explicit approximation of $h$. By logarithmic Sobolev and Csiszár-Kullback inequalities, for large values of $t, h(t, \cdot)$ converges to

$$
h_{\infty}(x):=\frac{e^{-\frac{|x|^{2}}{2 \kappa_{\omega}}}}{\left(2 \pi \kappa_{\omega}\right)^{1 / 2}},
$$

and one can even give a rate of convergence in $L^{1}(\mathbb{R})$ as follows.

LEMMA 2.1. With the above notations, if $h$ is a nonnegative solution of (2.10) with initial data $h_{0}$ such that $\int_{\mathbb{R}} h_{0} d x=1$ and $\int_{\mathbb{R}} h_{0} \log \left(h_{0} / h_{\infty}\right) d x<\infty$, then $\left\|h(t, \cdot)-h_{\infty}\right\|_{L^{1}(\mathbb{R})}=O\left(e^{-t}\right)$ as $t \rightarrow \infty$.

Proof. This result is standard, see [6, 44], but crucial for our approach. By density, it is enough to prove it for smooth solutions. The proof is based on the logarithmic Sobolev inequality,

$$
\int_{\mathbb{R}^{d}} h \log \left(\frac{h}{h_{\infty}}\right) d x \leq \frac{\kappa_{\omega}}{2} \int_{\mathbb{R}^{d}} h\left|\left(\log \left(\frac{h}{h_{\infty}}\right)\right)_{x}\right|^{2} d x,
$$

and on the Csiszár-Kullback inequality,

$$
\left\|h-h_{\infty}\right\|_{L^{1}\left(\mathbb{R}^{d}\right)}^{2} \leq \frac{1}{4} \int_{\mathbb{R}^{d}} h \log \left(\frac{h}{h_{\infty}}\right) d x
$$

Since $\frac{d}{d t} \int_{\mathbb{R}^{d}} h \log \left(\frac{h}{h_{\infty}}\right) d x=-\kappa_{\omega} \int_{\mathbb{R}^{d}} h\left|\log \left(\frac{h}{h_{\infty}}\right)_{x}\right|^{2} d x$, the conclusion easily follows using a Gronwall estimate.

Hence, up to an error of the order of $1 / R$, we can replace $h(t, x)$ by $h_{\infty}(x)$ in the ansatz for $U$. Summarizing our formal computations, we have found that

$$
u(t, x)=\mathrm{U}(t, x ; z)=\left(g_{\omega}(z)-\frac{x}{\kappa_{\omega} R} g_{\omega}^{(1)}(z)\right) h_{\infty}(x)(1+o(1))
$$

with $R=e^{t}$ and $z=R x-\frac{1}{2}\left(R^{2}-1\right) A(\omega)$. By undoing the change of variables (2.3), we obtain in the original variables the following asymptotic expansion

$$
f(t, x)=\left[g_{\omega}(x-\omega t)-\frac{x-c(\omega) t}{\kappa_{\omega} \sqrt{1+2 t}} g_{\omega}^{(1)}(x-\omega t)\right]_{7}^{h_{\infty}\left(\frac{x-c(\omega) t}{\sqrt{1+2 t}}\right)} \frac{\sqrt{1+2 t}}{\sqrt{1+2}}(1+o(1))
$$


as $t \rightarrow \infty$. However, this computation is formal in the sense that we have not proved that the remainder terms can be bounded uniformly in $t$. Actually the estimates are more subtle and the effective diffusion coefficient $\kappa_{\omega}$ enters in our analysis not only for studying the convergence of $h$ towards $h_{\infty}$, but also in the effective rate of convergence of $u$ towards $u_{\infty}$. This will be made clear in Theorem 2.4 below.

Before explaining how a rate of convergence can be rigorously determined, let us state an expression of $\kappa_{\omega}$ which is standard in homogenization theory and turns out to be useful in practical computations.

LEMMA 2.2. Let $\chi$ be the unique periodic solution of

$$
\chi^{\prime \prime}-\left(\psi^{\prime}+\omega\right) \chi^{\prime}=\psi^{\prime}+c(\omega)
$$

such that $\int_{0}^{1} \chi d z=0$. Then

$$
\kappa_{\omega}=\int_{0}^{1}\left|1+\chi^{\prime}\right|^{2} g_{\omega} d z>0
$$

As a consequence, we have $\kappa_{\omega_{\mid \omega=0}}=\left(\int_{0}^{1} e^{\psi} d z \int_{0}^{1} e^{-\psi} d z\right)^{-1}<1$ and $\lim _{\omega \rightarrow \infty} \kappa_{\omega}=1$. Proof. The expression of $\kappa_{\omega}$ is adapted from [26]. For completeness, we give a sketch of the proof. First of all, the function $\chi$ exists and is uniquely defined, as the minimum of the strictly convex functional $\chi \mapsto \int_{0}^{1}\left(\frac{1}{2}\left|\chi^{\prime}\right|^{2}+\left[\psi^{\prime}+c(\omega)\right] \chi\right) e^{-\omega x-\psi(x)} d x$ on the space $\left\{\chi \in H_{\text {per }}^{1}(0,1): \int_{0}^{1} \chi d z=0\right\}$. The only property of $\chi$ that we shall use is that for any smooth test function $f$,

$$
\int_{0}^{1}\left(\psi^{\prime}+c\right) f d z=\int_{0}^{1} \chi\left(f^{\prime}+\left(\psi^{\prime}+\omega\right) f\right)^{\prime} d z
$$

from which it follows that

$$
\kappa_{\omega}=1-2 \int_{0}^{1} \chi\left(g_{\omega}\right)_{z} d z-\int_{0}^{1} \chi\left(\psi^{\prime}+c\right) g_{\omega} d z
$$

using (2.8). A few integrations by parts and the fact that $\left(g_{\omega}\right)_{z}+\omega g_{\omega}+\psi^{\prime} g_{\omega}=A(\omega)$ is constant allow to prove $(2.13)$.

The limit case $\omega \rightarrow 0$ follows from the observation that $\left(\chi^{\prime} e^{-\psi}\right)^{\prime}=\psi^{\prime} e^{-\psi}$. An asymptotic expansion in terms of $1 / \omega$ shows the limit as $\omega \rightarrow \infty$. Duhamel's formula gives the positivity of $\kappa_{\omega}$. See [14] for more details.

The first goal of this paper is to rigorously establish (2.12). More precisely, under some simplifying assumptions, we will prove that $\mathrm{U}(t, x ; z)-g_{\omega}(z) h_{\infty}(x)$ converges to 0 , at an exponential rate. The corresponding result is stated in Theorem 2.7. We shall only sketch the main steps of the proof, before focusing on the estimates of the best constants in the key inequality for our approach.

First step: The structure of (2.7) suggests to introduce the notations

$$
\begin{aligned}
& \mathrm{L}_{0} \mathrm{U}:=\mathrm{U}_{z z}+\left(\left(\omega+\psi^{\prime}(z)\right) \mathrm{U}\right)_{z} \\
& \mathrm{~L}_{1} \mathrm{U}:=\left(2 \mathrm{U}_{z z}+\left(\psi^{\prime}(z)+c\right) \mathrm{U}\right)_{x} \\
& \mathrm{~L}_{2} \mathrm{U}:=\mathrm{U}_{x x}+(x \mathrm{U})_{x}-\mathrm{U}_{t} \\
& \mathrm{LU}:=-\left(R^{2} \mathrm{~L}_{0} \mathrm{U}+R \mathrm{~L}_{1} \mathrm{U}+\mathrm{L}_{2} \mathrm{U}\right)
\end{aligned}
$$


and $\mathrm{U}:=\mathrm{U}_{0}+R^{-1} \mathrm{U}_{1}+R^{-2} \mathrm{U}_{2}$,

$$
\begin{aligned}
& \mathrm{U}_{0}(t, x ; z):=g_{\omega}(z) h(t, x), \\
& \mathrm{U}_{1}(t, x ; z):=g_{\omega}^{(1)}(z) h_{x}(t, x), \\
& \mathrm{U}_{2}(t, x ; z):=g_{\omega}^{(2)}(z) h_{x x}(t, x),
\end{aligned}
$$

where $g_{\omega}$ and $g_{\omega}^{(1)}$ are defined as above respectively by (2.4) and (2.8), and $g_{\omega}^{(2)}$ solves

$$
\left(g_{\omega}^{(2)}\right)_{z z}+\left(\left(\omega+\psi^{\prime}(z)\right) g_{\omega}^{(2)}\right)_{z}+2\left(g_{\omega}^{(1)}\right)_{z}+\left(\psi^{\prime}(z)+c\right) g_{\omega}^{(1)}+\left(1-\kappa_{\omega}\right) g_{\omega}=0 .
$$

A careful computation shows that,

$$
\mathrm{LU}=\frac{1}{R}\left(\mathrm{~L}_{1} \mathrm{U}_{2}+\mathrm{L}_{2} \mathrm{U}_{1}\right)+\frac{1}{R^{2}} \mathrm{~L}_{2} \mathrm{U}_{2} .
$$

It turns out that $u(t, x)=\mathrm{U}(t, x ; z)$ with $R=R(t)=e^{t}, z=R x-\frac{1}{2}\left(R^{2}-1\right) A(\omega)$ is a solution of (2.6) if and only if $\mathrm{LU}=0$.

Second step: Consider the Gaussian function $h_{\infty}$ as in Lemma 2.1 and define

$$
\mathrm{U}_{\infty}:=\frac{1}{Z(t)}\left(\mathrm{U}_{\infty, 0}+R^{-1} \mathrm{U}_{\infty, 1}+R^{-2} \mathrm{U}_{\infty, 2}\right),
$$

with $z=e^{t} x-\frac{1}{2}\left(e^{2 t}-1\right) A(\omega)$ and $\mathrm{U}_{\infty, 0}(t, x ; z):=g_{\omega}(z) h_{\infty}(x), \mathrm{U}_{\infty, 1}(t, x ; z):=$ $g_{\omega}^{(1)}(z) h_{\infty, x}(x) \chi\left(e^{-t} x\right), \mathrm{U}_{\infty, 2}(t, x ; z):=g_{\omega}^{(2)}(z) h_{\infty, x x}(x) \chi\left(e^{-t} x\right)$, for some smooth truncation function $\chi$ which takes values 1 in a neighborhood of 0 and 0 outside of a ball. The role of $\chi$ is to enforce the positivity of $U_{\infty}$. The coefficient $Z$ is determined in such a way that, with $R=R(t)=e^{t}$,

$$
\int_{\mathbb{R}} \mathrm{U}_{\infty}\left(t, x ; R x-\frac{1}{2}\left(R^{2}-1\right) A(\omega)\right) d x=1 .
$$

Since $\mathrm{U}_{\infty}$ is only an approximate solution, we have

$$
\mathrm{LU}_{\infty}=\frac{\dot{Z}}{Z} \mathrm{U}_{\infty}+\frac{1}{R} \mathrm{~F}
$$

where $\mathrm{F} / \mathrm{U}_{\infty}$ is a polynomial of order four in $x$, with bounded coefficients depending on $t, x$ and $z$. Let us define

$$
u_{\infty}(t, x):=\mathrm{U}_{\infty}\left(t, x ; e^{t} x-\frac{1}{2}\left(e^{2 t}-1\right) A(\omega)\right)
$$

and

$$
\mathrm{f}(t, x):=\mathrm{F}\left(t, x ; e^{t} x-\frac{1}{2}\left(e^{2 t}-1\right) A(\omega)\right) .
$$

We will adapt the entropy/entropy production method sketched in the proof of Lemma 2.1. For this purpose, we first compute the derivative of the relative entropy with respect to $u_{\infty}$ as follows.

Lemma 2.3. Let $u$ be a solution of (2.6). Then

$$
\frac{d}{d t} \int_{\mathbb{R}} u \log \left(\frac{u}{u_{\infty}}\right) d x=-\int_{\mathbb{R}}\left|\left(\log \left(\frac{u}{u_{\infty}}\right)\right)_{x}\right|^{2} u d x+\frac{\dot{Z}}{Z}+e^{-t} \int_{\mathbb{R}} \frac{\mathrm{f}}{u_{\infty}} u d x
$$


Proof. The functions $u$ and $u_{\infty}$ respectively solve the equations

$$
u_{t}=u_{x x}+\left(\varphi^{\prime}(t, x) u\right)_{x}
$$

and

$$
\left(u_{\infty}\right)_{t}=\left(u_{\infty}\right)_{x x}+\left(\varphi^{\prime}(t, x) u_{\infty}\right)_{x}-\frac{\dot{Z}}{Z} u_{\infty}+e^{-t} \mathrm{f}
$$

for some function $\varphi$. The result follows by writing

$$
\frac{d}{d t} \int_{\mathbb{R}} u \log \left(\frac{u}{u_{\infty}}\right) d x=\int_{\mathbb{R}}\left[1+\log \left(\frac{u}{u_{\infty}}\right)\right] u_{t} d x-\int_{\mathbb{R}} \frac{u}{u_{\infty}}\left(u_{\infty}\right)_{t} d x
$$

and integrating by parts.

Third step: In the next step towards the justification of (2.12), we link the relative entropy $\int_{\mathbb{R}} u \log \left(u / u_{\infty}\right) d x$ with the relative Fisher information $\int_{\mathbb{R}}\left|\left(\log \left(u / u_{\infty}\right)\right)_{x}\right|^{2} u d x$ by a logarithmic Sobolev inequality. The estimate differs here from the formal approach, in the sense that the optimal constant in the homogenized limit is not the constant of the logarithmic Sobolev inequality in the limit case.

THEOREM 2.4. Let $u_{\infty}^{\varepsilon}(x):=c_{\varepsilon} e^{-\phi(x / \varepsilon)-|x|^{2} /\left(2 \kappa_{\omega}\right)} d x$, where $\phi$ is a $C^{2}$ function on $[0,1)$ and $c_{\varepsilon}$ is chosen such that $\int_{\mathbb{R}} u_{\infty}^{\varepsilon} d x=1$. For any $\varepsilon>0$, there exists a positive constant $\mathcal{K}_{\varepsilon}$ such that, for any nonnegative $u \in L^{1}(\mathbb{R})$ satisfying $\int_{\mathbb{R}} u d x=1$,

$$
\int_{\mathbb{R}} u \log \left(\frac{u}{u_{\infty}^{\varepsilon}}\right) d x \leq \mathcal{K}_{\varepsilon} \int_{\mathbb{R}}\left|\left(\log \left(\frac{u}{u_{\infty}^{\varepsilon}}\right)\right)_{x}\right|^{2} u d x .
$$

Moreover, $\lim \sup _{\varepsilon \rightarrow 0} \mathcal{K}_{\varepsilon}=: \mathrm{k} / 2$ satisfies $\kappa_{\omega} / \mathrm{K} \leq \mathrm{k} \leq \kappa_{\omega} \max _{[0,1]} g_{\omega} \cdot\left(\min _{[0,1]} g_{\omega}\right)^{-1}$ where $\mathrm{K}^{-1}=\int_{0}^{1} g_{\omega} d z \int_{0}^{1} g_{\omega}^{-1} d z$, and $\lim _{\omega \rightarrow 0} \mathrm{~K} / \kappa_{\omega}=1$.

A more precise statement, in connection with generalized Poincaré inequalities will be given in Theorem 4.1. The proof of this type of inequalities is the main purpose of this paper. However, among various functional inequalities, logarithmic Sobolev inequalities are especially interesting because of Lemma 2.3. Such an idea has been used for Brownian ratchets in $[9,24]$ and in case of diffusions with source terms in [23]. The upper estimate is a Holley-Stroock estimate and will be proved in details in Lemma 5.2. The limit of $\mathrm{K} / \kappa_{\omega}$ as $\omega \rightarrow 0$ has already been established in Lemma 2.2. Using Theorem 2.4 with $\phi:=-\log g_{\omega}$, where $g_{\omega}$ is given in terms of $\psi$ by (2.4), we obtain the following result.

Corollary 2.5. Assume that $\psi$ is $C^{2}$, periodic. With the notations of Theorem 2.4, there exists a function $t \mapsto C(t)$, with $\lim _{t \rightarrow \infty} C(t)=\mathrm{k} / 2$, which is positive, finite for any $t>0$, such that, for any $u \in L^{1}(\mathbb{R})$,

$$
\int_{\mathbb{R}} u \log \left(\frac{u}{u_{\infty}}\right) d x \leq C(t) \int_{\mathbb{R}}\left|\left(\log \left(\frac{u}{u_{\infty}}\right)\right)_{x}\right|^{2} u d x .
$$

Fourth step: Based on Lemma 2.3 and Corollary 2.5, the last step amounts to control the convergence of $u$ to $u_{\infty}$. Hence, we need to control the two source terms in (2.15). A direct computation, whose proof is left to the reader, shows that 
LEMma 2.6. With the above notations, if $u_{\infty}$ is given by (2.14), then

$$
\limsup _{t \rightarrow+\infty} e^{t} \frac{\dot{Z}(t)}{Z(t)}<\infty
$$

To control the second source term in (2.15), $\int_{\mathbb{R}} u_{\infty}^{-1} \mathrm{f} u d x$, we will assume that

$$
\limsup _{t \rightarrow+\infty} \int_{\mathbb{R}}|x|^{4} u(t, x) d x<\infty
$$

if $u$ is a solution of (2.6). We expect that this property is true for a large class of solutions, but have managed to prove it only under additional technical assumptions, by constructing a super-solution. A proof based on Hermite functions is given in an Appendix to this paper, but there is definitely space for improvements on this point.

Summarizing, we have obtained the following result.

Theorem 2.7. Let $d=1, \omega>0$, and assume that $\psi$ is $C^{2}$, periodic. Consider a solution $u$ of (2.6) and assume that (2.16) holds. With the notations of Theorem 2.4, for any $\delta>0$, we have

$$
\limsup _{t \rightarrow \infty} e^{(\min (1,1 / \mathrm{k})-\delta)) t}\left\|u(t)-u_{\infty}(t)\right\|_{L^{1}(\mathbb{R})}<\infty .
$$

Let $f_{\infty}(t, x):=R^{-1} u_{\infty}(\log R,(x-c t \mathrm{e}) / R)$, with $R(t)=\sqrt{1+2 t}$. We can also state a result on the intermediate asymptotics of the solutions of (1.1), using the change of coordinates (2.3).

Corollary 2.8. Under the assumptions of Theorem 2.7, if $f$ is a solution of (1.1) and if (2.16) holds, then for any $\delta>0$, we have

$$
\limsup _{t \rightarrow \infty} t^{(\min (1,1 / \mathrm{k})-\delta)) / 2}\left\|f(t)-f_{\infty}(t)\right\|_{L^{1}(\mathbb{R})}<\infty .
$$

It is worth noticing that as soon as $\mathrm{k}>1$, the rate of convergence in $L^{1}$ is governed by the logarithmic Sobolev inequality of Corollary 2.5. We know that $\mathrm{K} / \kappa_{\omega} \leq \mathrm{k}$, $\lim _{\omega \rightarrow 0} \mathrm{~K} / \kappa_{\omega}=1$ and at least for $\psi(x)=\sin (2 \pi x)$, we numerically observe that $\mathrm{K} / \kappa_{\omega}>1$ for any $\omega>0$. See [14] for more details.

3. Entropy methods and homogenization of functional inequalities. As already mentioned, our approach relies on entropy methods based on a logarithmic Sobolev inequality. We shall actually study Poincaré and logarithmic Sobolev inequalities, and a whole family of generalized Poincaré inequalities which interpolates between the usual Poincaré inequality and logarithmic Sobolev inequalities, see $[10,5,34,4,17,9]$. We will establish the expression of the sharp constants for such inequalities in the limit $\varepsilon \rightarrow 0$ and show that the large scale behavior of the solution is given by a Fokker-Planck equation with a modified diffusion. The method applies to much more general equations, and drift forces which are not of the form $\nabla\left(\phi(x / \varepsilon)+|x|^{2} / 2\right)$, but for sake of simplicity, we will only consider the case of Equation (1.2).

The strategy of entropy methods is quite simple. Let

$$
u_{\infty}^{\varepsilon}(x):=M \frac{e^{-\frac{1}{2}|x|^{2}-\phi(x / \varepsilon)}}{\int_{\mathbb{R}^{d}} e^{-\frac{1}{2}|z|^{2}-\phi(z / \varepsilon)} d z}
$$


be the unique stationary solution of (1.2) with mass $M=\int_{\mathbb{R}^{d}} u^{\varepsilon}(t, x) d x$ (which is always independent of $t$ ), and compute the time evolution of the convex entropy $\mathrm{E}_{\varepsilon}^{(p)}\left[u^{\varepsilon}\right]$ of the solution of $(1.2)$ as

$$
\frac{d}{d t} \mathrm{E}_{\varepsilon}^{(p)}\left[u^{\varepsilon}(t, \cdot)\right]:=-\mathrm{I}_{\varepsilon}^{(p)}\left[u^{\varepsilon}(t, \cdot)\right],
$$

where

$$
\mathrm{E}_{\varepsilon}^{(p)}[u]:=\frac{1}{p-1} \int_{\mathbb{R}^{d}}\left[\left(\frac{u}{u_{\infty}^{\varepsilon}}\right)^{p}-1-p\left(\frac{u}{u_{\infty}^{\varepsilon}}-1\right)\right] u_{\infty}^{\varepsilon} d x
$$

and

$$
\mathrm{I}_{\varepsilon}^{(p)}[u]:=p \int_{\mathbb{R}^{d}}\left(\frac{u}{u_{\infty}^{\varepsilon}}\right)^{p-2}\left|\nabla\left(\frac{u}{u_{\infty}^{\varepsilon}}\right)\right|^{2} u_{\infty}^{\varepsilon} d x
$$

for any $p \in(1,2]$. We will prove that, for any sufficiently smooth function $u$,

$$
\frac{4}{p} \mathcal{C}_{\varepsilon}^{(p)} \mathrm{E}_{\varepsilon}^{(p)}[u] \leq \mathrm{I}_{\varepsilon}^{(p)}[u]
$$

for some positive constant $\mathcal{C}_{\varepsilon}^{(p)}$, thus providing a exponential rate of decay of $t \mapsto$ $\mathrm{E}_{\varepsilon}^{(p)}\left[u^{\varepsilon}(t, \cdot)\right]$, which by the generalized Csiszár-Kullback inequality, see e.g. [9, 44], controls $\left\|u^{\varepsilon}(t, \cdot)-u_{\infty}^{\varepsilon}\right\|_{L^{p}\left(\mathbb{R}^{d}, u_{\infty}^{\varepsilon} d x\right)}$. The rate is sharp if the constant is optimal.

The main purpose of this paper is to understand the limit of $\mathcal{C}_{\varepsilon}^{(p)}$ as $\varepsilon \rightarrow 0$. Notice that the case $p=2$ in (3.1) corresponds to the Poincaré inequality (with respect to the measure $u_{\infty}^{\varepsilon} d x$ ), while in the limit case corresponding to $p \rightarrow 1, \mathrm{E}_{\varepsilon}^{(p)}[u]$ converges to

$$
\mathrm{E}_{\varepsilon}^{(1)}[u]:=\int_{\mathbb{R}^{d}} u \log \left(\frac{u}{u_{\infty}^{\varepsilon}}\right) d x
$$

and (3.1) with $p=1$ is a logarithmic Sobolev inequality. Inequality (3.1) will be referred to as the generalized Poincaré inequality or the convex Sobolev inequality following the definition of W. Beckner in [10] (in the Gaussian case), and later generalized in [6]. Also see [17, 34] for related issues.

The main tools of our approach are variational. We perform a detailed analysis of minimizing sequences. The difficulty comes from the fact that equality cases are sometimes achieved only by trivial functions, e.g. constant functions in the case of a Gaussian weight. E. Carlen and M. Loss proved in [16] that equality in the Euclidean logarithmic Sobolev inequalities, that is for Lebesgue's measure on $\mathbb{R}^{d}$, occurs for and only for Gaussian functions, which make simultaneously the entropy and the energy terms equal to zero. In some cases, this can also be seen as a consequence of the Bakry-Emery method, see [43], but this is not the case in the present framework. Hence, one has to carry a detailed analysis of the convergence and handle possible lacks of compactness.

Although not surprising from the point of view of homogenization theory, our estimates differ by several aspects of standard problems which have been abundantly treated in the literature. For instance, we deal with non compact domains, in functional spaces with oscillatory measures and determine sharp constants even in cases where there is no nontrivial solution of the Euler-Lagrange equations associated to 
the corresponding variational problem. As far as we know, tools of homogenization theory have not been used much in the framework of logarithmic Sobolev inequalities and semi-group theory. We think that this is an extremely interesting field with applications of large interest.

Let us start with an observation that provides us with a simplifying assumption. Since adding a constant to the potential does not produce any change for the solution of (1.2), we can assume that

$$
\int_{\mathbb{T}^{d}} e^{-\phi(y)} d y=1
$$

As a consequence, we observe that the function

$$
\tau(z):=\int_{\partial \mathbb{T}^{d}} e^{-\phi(y+z)} d \sigma_{1}(y),
$$

where $d \sigma_{1}(y)=y \cdot \nu(y) d \sigma_{0}(y)$, is such that, by (3.2) and because of the periodicity of $\phi$,

$$
\int_{\mathbb{T}^{d}} \tau(z) d z=\int_{\partial \mathbb{T}^{d}} d \sigma_{1}(y) \int_{\mathbb{T}^{d}} e^{-\phi(y+z)} d z=d
$$

Here we denote by $\nu(y)$ the unit vector at $y \in \partial \mathbb{T}^{d}$ which is orthogonal to $\partial \mathbb{T}^{d}$ and pointing outwards, and by $d \sigma_{0}$ the measure induced by Lebesgue's measure on $\partial \mathbb{T}^{d}$. Let $z_{0} \in \mathbb{T}^{d}$ be such that $\tau\left(z_{0}\right)=d$. Such a $z_{0}$ exists if, for instance, $\tau$ is continuous and periodic. We shall from now on assume that $z_{0}=0$, so that

$$
\int_{\partial \mathbb{T}^{d}} e^{-\phi(y)} d \sigma_{1}(y)=d
$$

As a consequence,

$$
\int_{\mathbb{T}^{d}} y \cdot \nabla_{y}\left(e^{-\phi(y)}\right) d y=0
$$

Before going to precise statements, let us make a formal asymptotic expansion which explains the qualitative behavior of the solutions. Assume that the solution of (1.2) can be written as

$$
u^{\varepsilon}(t, x)=u^{(0)}\left(t, x, \frac{x-x_{0}}{\varepsilon}\right)+\varepsilon u^{(1)}\left(t, x, \frac{x-x_{0}}{\varepsilon}\right)+\varepsilon^{2} u^{(2)}\left(t, x, \frac{x-x_{0}}{\varepsilon}\right)+O\left(\varepsilon^{3}\right)
$$

for some $x_{0} \in \mathbb{T}^{d}$. By $O\left(\varepsilon^{3}\right)$, we mean that the remainder term is of lower order as well as its derivatives with respect to $x$. Choosing $x_{0} \neq 0$ is equivalent to shift $\phi$ of $x_{0}$. Hence, by an appropriate choice of $x_{0}$, we can impose that (3.2) holds. We shall also assume that all functions $y \mapsto u^{(i)}(t, x, y)$ are periodic for any fixed $t>0$, $x \in \mathbb{R}^{d}$. Let

$$
v^{(i)}(t, x, y):=u^{(i)}(t, x, y) e^{\phi(y)}, \quad i=1,2,3 .
$$

Injecting this ansatz for $u^{\varepsilon}(t, x)$ in (1.2) and formally solving the equation order by order in $\varepsilon$, we find that the functions $u^{(i)}, i=1,2,3$, solve the following equations. 
$\underline{\text { At } \operatorname{order} \varepsilon^{-2}:}$

$$
\Delta_{y} u^{(0)}+\nabla_{y} \cdot\left(u^{(0)} \nabla_{y} \phi(y)\right)=0
$$

that is $v^{(0)}$ does not depend on $y$. As a consequence, we have

$$
\nabla_{y} u^{(0)}=-\nabla_{y} \phi(y) u^{(0)} .
$$

We may also observe that

$$
\int_{\mathbb{R}^{d}} u^{\varepsilon}(t, x) d x=\int_{\mathbb{R}^{d}} v^{(0)}(t, x) d x+O(\varepsilon),
$$

so that $M_{\varepsilon}:=\int_{\mathbb{R}^{d}} v^{(0)}(t, x) d x=M+O(\varepsilon)$ as $\varepsilon \rightarrow 0$.

At $\operatorname{order} \varepsilon^{-1}$ :

$$
\Delta_{y} u^{(1)}+\nabla_{y} \cdot\left(u^{(1)} \nabla_{y} \phi(y)\right)=-\nabla_{x} \cdot\left(2 \nabla_{y} u^{(0)}+\nabla_{y} \phi(y) u^{(0)}\right)=\nabla_{y} \phi(y) \cdot \nabla_{x} u^{(0)},
$$

that is

$$
\nabla_{y} \cdot\left(e^{-\phi(y)}\left(\nabla_{y} v^{(1)}+\nabla_{x} v^{(0)}\right)\right)=0
$$

which amounts to write that

$$
v^{(1)}(t, x, y)=\nabla_{x} v^{(0)}(t, x) \cdot w(t, y)
$$

where $w(t, y)=\left(w_{j}(t, y)\right)_{j=1}^{d}$ is a solution of the so-called cell equation, that is $w_{j}$ is, up to an arbitrary constant, an $y$-periodic solution of

$$
\nabla_{y} \cdot\left(e^{-\phi(y)}\left(\nabla_{y} w_{j}+\mathrm{e}_{j}\right)\right)=0
$$

and $\mathrm{e}_{j}$ is the unit vector with coordinates $\left(\delta_{i j}\right)_{i=1}^{d}$, where $\delta_{i j}$ stands for Kronecker's symbol. A solution of the cell equation is given by

$$
\frac{\partial w_{j}}{\partial y_{i}}+\delta_{i j}=c_{i j} e^{\phi(y)}
$$

for some constant $\mathrm{c}_{i j}$ to be determined. Using the periodicity of $w_{j}$ with respect to $y_{i}$, an integration on $\mathbb{T}^{d}$ gives

$$
\begin{gathered}
\delta_{i j}=\mathrm{c}_{i j} \int_{\mathbb{T}^{d}} e^{\phi(y)} d y, \\
\left(\mathrm{c}_{i j}\right)_{i=1}^{d}=\frac{\mathrm{e}_{j}}{\int_{\mathbb{T}^{d}} e^{\phi(y)} d y} .
\end{gathered}
$$

Thus we have obtained that

$$
\nabla_{y} w_{j}=\left[\frac{e^{\phi}}{\int_{\mathbb{T}^{d}} e^{\phi(y)} d y}-1\right] \mathrm{e}_{j} .
$$


By the Maximum Principle, it is not difficult to see that $w$ is uniquely defined if we further assume that

$$
\int_{\mathbb{T}^{d}} w d y=0
$$

This means

$$
u^{(1)}(t, x, y)=\nabla_{x} v^{(0)}(x) \cdot w(t, y) e^{-\phi(y)}
$$

At order $\varepsilon^{0}=1$ :

$$
\begin{aligned}
u_{t}^{(0)}=\nabla_{y} \cdot\left(\nabla_{y} u^{(2)}+u^{(2)} \nabla_{y} \phi(y)\right) & +\nabla_{x} \cdot\left(\nabla_{x} u^{(0)}+x u^{(0)}\right) \\
& +\nabla_{x} \cdot\left(2 \nabla_{y} u^{(1)}+\nabla_{y} \phi(y) u^{(1)}\right)+y \cdot \nabla_{y} u^{(0)}
\end{aligned}
$$

that is

$$
\begin{aligned}
u_{t}^{(0)}=\nabla_{y} \cdot\left(e^{-\phi(y)} \nabla_{y} v^{(2)}\right) & +\nabla_{x} \cdot\left(\nabla_{x} v^{(0)}+x v^{(0)}\right) e^{-\phi(y)} \\
& +\nabla_{x} \cdot\left(2 \nabla_{y} u^{(1)}+\nabla_{y} \phi(y) u^{(1)}\right)+y v^{(0)} \cdot \nabla_{y}\left(e^{-\phi(y)}\right) .
\end{aligned}
$$

We do not need to solve the equation for $v^{(2)}$ but can simply examine the solvability condition which goes as follows. Formally integrate with respect to $y \in \mathbb{T}^{d}$ to get

$$
\begin{aligned}
{\left[v_{t}^{(0)}-\nabla_{x} \cdot\right.} & \left.\left(\nabla_{x} v^{(0)}+x v^{(0)}\right)\right] \int_{\mathbb{T}^{d}} e^{-\phi(y)} d y \\
& =-\nabla_{x} \cdot \int_{\mathbb{T}^{d}} v^{(1)}(t, x, y) \nabla_{y}\left(e^{-\phi(y)}\right) d y+\int_{\mathbb{T}^{d}} y v^{(0)} \cdot \nabla_{y}\left(e^{-\phi(y)}\right) d y \\
& =\nabla_{x} \cdot \int_{\mathbb{T}^{d}} \nabla_{y} v^{(1)}(t, x, y) e^{-\phi(y)} d y \\
& =\sum_{i, j=1}^{d} \frac{\partial^{2} v^{(0)}}{\partial x_{i} \partial x_{j}} \int_{\mathbb{T}^{d}} \frac{\partial w_{j}}{\partial y_{i}} e^{-\phi(y)} d y \\
& =\Delta_{x} v^{(0)}\left[\frac{1}{\int_{\mathbb{T}^{d}} e^{\phi(y)} d y}-\int_{\mathbb{T}^{d}} e^{-\phi(y)} d y\right] \\
& =\Delta_{x} v^{(0)}\left[\frac{1}{\int_{\mathbb{T}^{d}} e^{\phi(y)} d y}-1\right]
\end{aligned}
$$

where we have used the fact that $v^{(0)}$ does not depend on $y$, the periodicity in $y$, an integration by parts, the condition (3.3), the solution of the cell equation (3.6), and the normalization condition (3.2). Notice that if (3.3) does not hold, we can still choose $x_{0}$ in (3.4) so that, in the second line,

$$
\int_{\mathbb{T}^{d}}\left(x_{0}+y\right) \cdot \nabla_{y}\left(e^{-\phi(y)}\right) d y=0 .
$$

The choice of $x_{0}$ in (3.4) is therefore determined by the solvability condition at order $\varepsilon^{0}=1$ in the formal asymptotic expansion. Let

$$
\mathrm{K}:=\frac{1}{\int_{\mathbb{T}^{d}} e^{\phi(y)} d y \int_{\mathbb{T}^{d}} e^{-\phi(y)} d y}=\frac{1}{\int_{\mathbb{T}^{d}} e^{\phi(y)} d y}
$$


and observe that $\mathrm{K} \leq 1$ since by Cauchy-Schwarz' inequality,

$$
1=\left(\int_{\mathbb{T}^{d}} 1 d y\right)^{2} \leq \int_{\mathbb{T}^{d}} e^{\phi(y)} d y \int_{\mathbb{T}^{d}} e^{-\phi(y)} d y .
$$

The equation satisfied by $v^{(0)}$ is

$$
v_{t}^{(0)}=\mathrm{K} \Delta v^{(0)}+\nabla \cdot\left(x v^{(0)}\right)
$$

The standard theory of the Fokker-Planck equations then shows that $v^{(0)}$ converges as $t \rightarrow \infty$ to

$$
v_{\infty}^{(0)}(x)=\frac{M}{(2 \pi \mathrm{K})^{d / 2}} e^{-\frac{|x|^{2}}{2 K}} .
$$

Moreover, with the notation (2.11), we observe that by Lemma 2.2,

$$
\kappa_{\omega_{\mid \omega=0}}=\mathrm{K} .
$$

Summarizing, we have obtained that the solution $u^{\varepsilon}(t, x)$ of $(1.2)$ can be written as

$$
u^{\varepsilon}(t, x)=\left(v^{(0)}(t, x)+\varepsilon \nabla_{x} v^{(0)}(t, x) \cdot w\left(t, \frac{x}{\varepsilon}\right)+O\left(\varepsilon^{2}\right)\right) e^{-\phi\left(\frac{x}{\varepsilon}\right)}
$$

where $w$ is a solution of the cell problem (3.5) and $v^{(0)}$ is a solution of the FokkerPlanck equation (3.8), with diffusion coefficient $\mathrm{K}$ given by (3.7), which converges to the Gaussian function $v_{\infty}^{(0)}$. Hence we have the following diagram:

$$
\begin{aligned}
& u^{\varepsilon}(t, x) \quad \underset{t \rightarrow \infty}{\stackrel{L^{1} \cap L^{2}}{\longrightarrow}} \quad u_{\infty}^{\varepsilon}(x)=M \frac{e^{-\frac{1}{2}|x|^{2}-\phi(x / \varepsilon)}}{\int_{\mathbb{R}^{d}} e^{-\frac{1}{2}|z|^{2}-\phi(z / \varepsilon)} d z} \underset{\varepsilon \rightarrow 0}{\stackrel{\text { two-scale }}{\longrightarrow}} \frac{M}{(2 \pi)^{d / 2}} e^{-\frac{|x|^{2}}{2}} e^{-\phi(y)} \\
& \|
\end{aligned}
$$

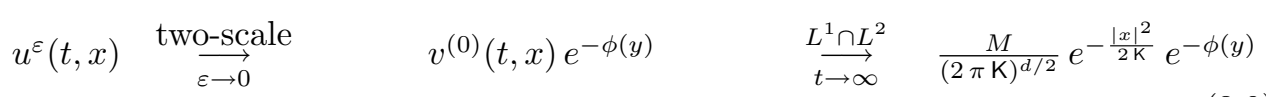

It is interesting to observe that the diagram does not commute, and that the homogenized problem, that is, the limit of $u^{\varepsilon}(t, x)$ as $\varepsilon \rightarrow 0$, behaves for large values of $t$ like the solutions of a modified Fokker-Planck equation (3.8) with diffusion coefficient $\mathrm{K}<1$. We shall see in Corollary 7.1 that, as $t \rightarrow \infty$, the rate of convergence in the first line is given by

$$
\left\|u^{\varepsilon}-u_{\infty}^{\varepsilon}\right\|_{L^{p}\left(\mathbb{R}^{d},\left(u_{\infty}^{\varepsilon}\right)^{1-p} d x\right)}=O\left(e^{-2 \mathcal{C}_{\varepsilon}^{(p)} t / p}\right)
$$

where $2 \mathcal{C}_{\varepsilon}^{(p)} / p$ converges to $\mathrm{K}$ as $\varepsilon \rightarrow 0$ for any $p \in(1,2]$. As $t \rightarrow \infty$, the rate of convergence in the second line is determined by

$$
\left\|v^{(0)}-v_{\infty}^{(0)}\right\|_{L^{p}\left(\mathbb{R}^{d},\left(v_{\infty}^{(0)}\right)^{1-p} d x\right)}=O\left(e^{-\mathrm{K} t / p}\right)
$$

for any $p \in(1,2]$. The goal of the rest of this paper is to establish the limit of $\mathcal{C}_{\varepsilon}^{(p)}$. 
4. Main result. On $\mathbb{R}^{d} \ni x$, let $\mu_{0}(x):=Z_{0}^{-1} e^{-|x|^{2} / 2}$ where $Z_{0}=(2 \pi)^{d / 2}$ is the normalized centered Gaussian function. For any $\varepsilon>0$, define

$$
\mu_{\varepsilon}(x):=Z_{\varepsilon}^{-1} e^{-\phi(x / \varepsilon)} \mu_{0}(x) \quad \text { with } \quad Z_{\varepsilon}=\int_{\mathbb{R}^{d}} e^{-\phi(x / \varepsilon)} \mu_{0}(x) d x .
$$

The function $\phi$ is a periodic function of $\mathbb{R}^{d}$ such that

$$
\phi(x)=\phi(\{x\}) \quad \forall x \in \mathbb{R}^{d}
$$

where $[x]$ is the unique element of $\mathbb{Z}^{d}$ such that $\{x\} \in[0,1)^{d} \approx \mathbb{T}^{d}$ and $\{x\}=x-[x]$.

To the measures $\left(\mu_{\varepsilon}\right)_{\varepsilon \geq 0}$, we associate the optimal Poincaré constants

$$
\mathcal{C}_{\varepsilon}^{(2)}:=\inf _{\substack{\int_{\mathbb{R}^{d}} u d \mu_{\varepsilon}=0 \\ 0 \neq u \in H^{1}\left(d \mu_{\varepsilon}\right)}} \frac{\int_{\mathbb{R}^{d}}|\nabla u|^{2} d \mu_{\varepsilon}}{\int_{\mathbb{R}^{d}}|u|^{2} d \mu_{\varepsilon}}
$$

for any $\varepsilon \geq 0$. Here the space $H^{1}\left(d \mu_{\varepsilon}\right)=H^{1}\left(\mathbb{R}^{d}, d \mu_{\varepsilon}\right)$ is the completion of $\mathcal{D}\left(\mathbb{R}^{d}\right)$ with respect to the norm $u \mapsto\left[\int_{\mathbb{R}^{d}}\left(|\nabla u|^{2}+|u|^{2}\right) d \mu_{\varepsilon}\right]^{1 / 2}$. We also define $\nu:=Z^{-1} e^{-\phi}$ with $Z=\int_{\mathbb{T}^{d}} e^{-\phi} d y$ and $H_{\mathrm{per}}^{1}\left(\mathbb{T}^{d}, d \nu\right)$ as the space of functions in $H_{\mathrm{loc}}^{1}\left(\mathbb{R}^{d}, d \nu\right)$ which only depend on $\{x\}$. Under Assumption (3.2), $Z=1$.

We can also define the sharp constant in the logarithmic Sobolev inequality by

$$
\mathcal{C}_{\varepsilon}^{(1)}:=\inf _{\substack{\nabla u \neq 0 d \mu_{\varepsilon} \text { a.e. } \\ u \in H^{1}\left(d \mu_{\varepsilon}\right)}} \frac{\int_{\mathbb{R}^{d}}|\nabla u|^{2} d \mu_{\varepsilon}}{\int_{\mathbb{R}^{d}}|u|^{2} \log \left(\frac{|u|^{2}}{\int_{\mathbb{R}^{d}}|u|^{2} d \mu_{\varepsilon}}\right) d \mu_{\varepsilon}}
$$

for any $\varepsilon \geq 0$, and the sharp constant in a family of generalized Poincaré inequalities which interpolates between the Poincaré and the logarithmic Sobolev inequalities

$$
\begin{aligned}
\mathcal{C}_{\varepsilon}^{(p)}:=(p-1) & \inf _{\nabla u} \neq 0 d \mu_{\varepsilon} \text { a.e. } \frac{\int_{\mathbb{R}^{d}}|\nabla u|^{2} d \mu_{\varepsilon}}{\int_{\mathbb{R}^{d}}|u|^{2} d \mu_{\varepsilon}-\left(\int_{\mathbb{R}^{d}}|u|^{2 / p} d \mu_{\varepsilon}\right)^{p}} \\
u & \in H^{1}\left(d \mu_{\varepsilon}\right)
\end{aligned}
$$

where $p \in(1,2)$ is a parameter. See $[4,5,6,8,9,10,17,34]$ for more details. We may observe that the above definitions are consistent in the sense that

$$
\lim _{p \rightarrow 1_{+}} \frac{1}{p-1}\left[\int_{\mathbb{R}^{d}}|u|^{2} d \mu_{\varepsilon}-\left(\int_{\mathbb{R}^{d}}|u|^{2 / p} d \mu_{\varepsilon}\right)^{p}\right]=\int_{\mathbb{R}^{d}}|u|^{2} \log \left(\frac{|u|^{2}}{\int_{\mathbb{R}^{d}}|u|^{2} d \mu_{\varepsilon}}\right) d \mu_{\varepsilon} .
$$

When $p \rightarrow 2$, one does not recover directly the definition of the Poincaré constant, since

$$
\lim _{p \rightarrow 2_{-}} \frac{1}{p-1}\left[\int_{\mathbb{R}^{d}}|u|^{2} d \mu_{\varepsilon}-\left(\int_{\mathbb{R}^{d}}|u|^{2 / p} d \mu_{\varepsilon}\right)^{p}\right]=\int_{\mathbb{R}^{d}}|u|^{2} d \mu_{\varepsilon}-\left(\int_{\mathbb{R}^{d}}|u| d \mu_{\varepsilon}\right)^{2}
$$

is not equal to $\int_{\mathbb{R}^{d}}|u|^{2} d \mu_{\varepsilon}-\left(\int_{\mathbb{R}^{d}} u d \mu_{\varepsilon}\right)^{2}$, but as already noted in [4], $\mathcal{C}_{\varepsilon}^{(2)}$ is equal to the Poincaré constant.

With $\operatorname{Osc}_{\mathbb{T}^{d}}(\phi):=\max _{\mathbb{T}^{d}} \phi-\min _{\mathbb{T}^{d}} \phi$, let

$$
\mathrm{k}:=\exp \left(-\mathrm{Osc}_{\mathbb{T}^{d}}(\phi)\right) .
$$


TheOrem 4.1. Assume that $\phi$ is a $C^{2}$ function on $\mathbb{T}^{d}$. With the above notations, for any $p \in(1,2]$,

$$
\lim _{\varepsilon \rightarrow 0_{+}} \mathcal{C}_{\varepsilon}^{(p)}=K \mathcal{C}_{0}^{(p)}
$$

Moreover, $\lim _{\varepsilon \rightarrow 0_{+}} \mathcal{C}_{\varepsilon}^{(1)} \in\left[\mathrm{k} \mathcal{C}_{0}^{(1)}, \mathrm{K} \mathcal{C}_{0}^{(1)}\right]$.

Here $\mathrm{K}$ is given by (3.7): $\mathrm{K}=1 / \int_{\mathbb{T}^{d}} e^{\phi(y)} d y$, and we observe that $\mathrm{K}<1$ as soon as $\phi$ is non trivial. In this paper, since we are dealing with the harmonic potential, or equivalently, with the Gaussian measure, the constant $\mathcal{C}_{0}^{(p)}$ is explicit: $\mathcal{C}_{0}^{(p)}=p / 2$, see [10], but our results are easy to generalize to other potentials which are uniformly strictly convex, up to bounded perturbations. As far as we know, it is an open question to determine whether $\lim _{\varepsilon \rightarrow 0_{+}} \mathcal{C}_{\varepsilon}^{(1)}=\mathrm{K} \mathcal{C}_{0}^{(1)}$ or not.

It remains to check that $\mathcal{C}_{\varepsilon}^{(p)}$ is the constant which appears in (3.1). If we assume that $M=1$, which is not a restriction because of the homogeneity, we may observe that, as a function of $\zeta$,

$$
\zeta \mapsto \frac{p-1}{\zeta^{p}} \mathrm{E}_{\varepsilon}^{(p)}\left[\zeta\left(v u_{\infty}^{\varepsilon}\right)^{2 / p}\right]=\int_{\mathbb{R}^{d}}|v|^{2} d \mu_{\varepsilon}+\frac{p-1}{\zeta^{p}}-\frac{p}{\zeta^{p-1}} \int_{\mathbb{R}^{d}}|v|^{2 / p} d \mu_{\varepsilon}
$$

achieves its minimum as a function of $\zeta>0$ for

$$
\zeta^{-1}=\int_{\mathbb{R}^{d}}|v|^{2 / p} d \mu_{\varepsilon}
$$

The result then follows since

$$
\mathrm{E}_{\varepsilon}^{(p)}\left[\zeta\left(v u_{\infty}^{\varepsilon}\right)^{2 / p}\right]=\frac{\zeta^{p}}{p-1}\left[\int_{\mathbb{R}^{d}}|v|^{2} d \mu_{\varepsilon}-\left(\int_{\mathbb{R}^{d}}|v|^{2 / p} d \mu_{\varepsilon}\right)^{p}\right]
$$

and

$$
\mathbf{I}_{\varepsilon}^{(p)}\left[\zeta\left(v u_{\infty}^{\varepsilon}\right)^{2 / p}\right]=\frac{4 \zeta^{p}}{p} \int_{\mathbb{R}^{d}}|\nabla v|^{2} d \mu_{\varepsilon}
$$

\section{Some preliminary results for the proof of Theorem 4.1.}

5.1. An upper estimate. With $\mathrm{e} \in S^{d-1}$, we use $u_{\mathrm{e}}(x)=x \cdot \mathrm{e}$, which is an eigenfunction associated to 1 , the first non-zero eigenvalue of $-\Delta+x \cdot \nabla$, as a test function. This gives a non explicit upper estimate for $\mathcal{C}_{\varepsilon}^{(p)}$ for any $\varepsilon>0$ and allows us to investigate the limit $\varepsilon \rightarrow 0$ :

$$
\begin{aligned}
& \lim _{\varepsilon \rightarrow 0} \int_{\mathbb{R}^{d}}\left|u_{\mathrm{e}}\right|^{2} d \mu_{\varepsilon}=\lim _{\varepsilon \rightarrow 0} \int_{\mathbb{R}^{d}}\left|\nabla u_{\mathrm{e}}\right|^{2} d \mu_{\varepsilon}=1, \\
& \lim _{\varepsilon \rightarrow 0} \int_{\mathbb{R}^{d}}\left|u_{\mathrm{e}}\right|^{2 / p} d \mu_{\varepsilon}=\frac{2^{1 / p}}{\sqrt{\pi}} \Gamma\left(\frac{1}{2}+\frac{1}{p}\right), \\
& \lim _{\varepsilon \rightarrow 0} \int_{\mathbb{R}^{d}}\left|u_{\mathrm{e}}\right|^{2} \log \left|u_{\mathrm{e}}\right|^{2} d \mu_{\varepsilon}=\log 2-2+\gamma \approx-0.729637,
\end{aligned}
$$

where $\gamma \approx 0.577216$ is Euler's constant. The function

$$
\kappa(p):=\frac{p-1}{1-\frac{2^{1 / p}}{\sqrt{\pi}} \Gamma\left(\frac{1}{2}+\frac{1}{p}\right)}, \quad p \in(1,2),
$$


is an increasing function on $(1,2)$ such that $\lim _{p \rightarrow 1_{+}} 1 / \kappa(p)=-\log 2+2-\gamma \approx 1.37054$ and $1 / \kappa(2)=1-\sqrt{2 / \pi} \approx 4.94767$.

Lemma 5.1. Assume that $\phi \in L^{\infty}\left(\mathbb{T}^{d}\right)$ satisfies (3.2). Then

$$
\lim _{\varepsilon \rightarrow 0} \mathcal{C}_{\varepsilon}^{(p)} \leq \kappa(p) .
$$

5.2. Perturbations of convex Sobolev inequalities. Perturbing the measure in the case of a Poincaré inequality is essentially trivial. In the case of the logarithmic Sobolev inequality, this has been done by R. Holley and D. Stroock in [29]. More general entropy functionals have been considered in [6], which cover all $\varphi$-entropies (see definition below). Also see [3, 17]. For completeness we give a simple proof of this result.

Assume that for some probability measure $d \mu$, the following convex Sobolev inequality holds

$$
\int\left[\varphi(u)-\varphi(\bar{u})-\varphi^{\prime}(\bar{u})(u-\bar{u})\right] d \mu \leq \mathcal{C}_{\varphi} \int \varphi^{\prime \prime}(u)|\nabla u|^{2} d \mu \quad \forall u \in H^{1}(d \mu) .
$$

Here we denote by $\bar{u}$ the average of $u$ with respect to $d \mu: \bar{u}:=\int u d \mu$. The left hand side is what we call a $\varphi$-entropy according to, for instance, [17]. If the inequality holds, then $\varphi$ has to be convex.

Assume next that $d \tilde{\mu}$ is a measure which is absolutely continuous with respect to $d \mu$ and such that

$$
e^{-b} d \mu \leq d \tilde{\mu} \leq e^{-a} d \mu \quad \mu \text { a.e. }
$$

for some constants $a, b \in \mathbb{R}$.

Lemma 5.2. Under the above assumptions, if $\varphi$ is a convex $C^{3}$ function with $\varphi^{\prime \prime}>0$ such that (5.1) holds, then

$$
\int\left[\varphi(u)-\varphi(\tilde{u})-\varphi^{\prime}(\tilde{u})(u-\tilde{u})\right] d \tilde{\mu} \leq \tilde{\mathcal{C}}_{\varphi} \int \varphi^{\prime \prime}(u)|\nabla u|^{2} d \tilde{\mu} \quad \forall u \in H^{1}(d \mu),
$$

where $\tilde{u}:=\int u d \tilde{\mu} / \int d \tilde{\mu}$ and $\tilde{\mathcal{C}}_{\varphi}:=e^{b-a} \mathcal{C}_{\varphi}$.

Proof. With the notation $\varphi^{\prime}=\frac{d \varphi}{d t}$, consider the function

$$
t \mapsto f(t):=\varphi(t)-t \varphi^{\prime}(t)+\varphi^{\prime}(t) \bar{u} .
$$

Its unique critical point is such that

$$
0=\frac{d}{d t}\left[\varphi(t)-t \varphi^{\prime}(t)+\varphi^{\prime \prime}(t) \bar{u}\right]=\varphi^{\prime \prime}(t)(\bar{u}-t),
$$

that is $t=\bar{u}$. By computing

$$
f^{\prime \prime}(\bar{u})=\varphi^{\prime \prime \prime}(t)(\bar{u}-t)_{\mid t=\bar{u}}-\varphi^{\prime \prime}(\bar{u})=-\varphi^{\prime \prime}(\bar{u})<0,
$$

we observe that $\bar{u}$ is the unique global maximum point of $f$. As a consequence,

$$
\begin{aligned}
& \int {[\varphi(u)-f(\bar{u})] d \mu } \\
&=\int\left[\varphi(u)-\varphi(\bar{u})-\varphi^{\prime}(\bar{u})(u-\bar{u})\right] d \mu \geq \int\left[\varphi(u)-\varphi(\tilde{u})-\varphi^{\prime}(\tilde{u})(u-\tilde{u})\right] d \mu \\
&=\int[\varphi(u)-f(\tilde{u})] d \mu .
\end{aligned}
$$


The result follows as a consequence of the following computation

$$
\begin{aligned}
e^{b} \mathcal{C}_{\varphi} \int \varphi^{\prime \prime}(u)|\nabla u|^{2} d \tilde{\mu} & \geq \mathcal{C}_{\varphi} \int \varphi^{\prime \prime}(u)|\nabla u|^{2} d \mu \\
& \geq \int\left[\varphi(u)-\varphi(\bar{u})-\varphi^{\prime}(\bar{u})(u-\bar{u})\right] d \mu \\
& \geq \int\left[\varphi(u)-\varphi(\tilde{u})-\varphi^{\prime}(\tilde{u})(u-\tilde{u})\right] d \mu \\
& \geq e^{a} \int\left[\varphi(u)-\varphi(\tilde{u})-\varphi^{\prime}(\tilde{u})(u-\tilde{u})\right] d \tilde{\mu}
\end{aligned}
$$

which completes the proof.

Lemma 5.2 applies in the case $\varphi(u)=\frac{u^{p}-1-p(u-1)}{p-1}$.

Corollary 5.3. With the above notations, if $\phi$ is bounded on $\mathbb{T}^{d}$, then for any $p \in[1,2]$,

$$
\mathcal{C}_{\varepsilon}^{(p)} \geq \frac{p}{2} e^{-\operatorname{Osc}(\phi)}
$$

5.3. Two scale convergence. Let us recall some standard results on the twoscale convergence, taken from [2]. We will consider the space $C^{\infty}\left(\mathbb{T}^{d}\right)$ of infinitely differentiable functions $u$ on $\mathbb{R}^{d}$ such that $u(x+k)=u(x)$ for any $x \in \mathbb{R}^{d}$ and any $k \in \mathbb{Z}^{d}$, and denote by $L^{2}\left(\mathbb{T}^{d}\right)$ and $H^{1}\left(\mathbb{T}^{d}\right)$ the corresponding Lebesgue and Sobolev spaces of periodic functions.

Proposition 5.4. Let $\Omega$ be an open set in $\mathbb{R}^{d}$. If $\left(u_{\varepsilon}\right)_{\varepsilon>0}$ is a bounded sequence in $L^{2}(\Omega)$, then there exists a subsequence of $\left(u_{\varepsilon}\right)_{\varepsilon>0}$, still denoted by $\left(u_{\varepsilon}\right)_{\varepsilon>0}$, and a function $u_{0} \in L^{2}\left(\Omega \times \mathbb{T}^{d}\right)$ such that

$$
\lim _{\varepsilon \rightarrow 0} \int_{\Omega} u_{\varepsilon}(x) \varphi\left(x, \frac{x}{\varepsilon}\right) d x=\iint_{\Omega \times \mathbb{T}^{d}} u_{0}(x, y) \varphi(x, y) d x d y,
$$

for all smooth $y$-periodic function $\varphi$. Moreover, $\left(u_{\varepsilon}\right)_{\varepsilon>0}$ weakly converges in $L^{2}(\Omega)$ to

$$
u_{*}(x):=\int_{\mathbb{T}^{d}} u_{0}(x, y) d y
$$

and

$$
\lim _{\varepsilon \rightarrow 0}\left\|u_{\varepsilon}\right\|_{L^{2}(\Omega)} \geq\left\|u_{0}\right\|_{L^{2}\left(\Omega \times \mathbb{T}^{d}\right)} \geq\left\|u_{*}\right\|_{L^{2}(\Omega)}
$$

Property (5.2) provides a definition of the two-scale convergence. The next result is taken from [2, Proposition 1.14].

Proposition 5.5. Let $\Omega$ be an open set in $\mathbb{R}^{d}$ and consider a sequence $\left(u_{\varepsilon}\right)_{\varepsilon>0}$ which weakly converges to $u_{*}$ in $H^{1}(\Omega)$. Then there exist a subsequence of $\left(u_{\varepsilon}\right)_{\varepsilon>0}$, still denoted $\left(u_{\varepsilon}\right)_{\varepsilon>0}$, which two-scale converges to $u_{*}$. Moreover, there exists a function $u_{1} \in L^{2}\left(\Omega, H^{1}\left(\mathbb{T}^{d}\right)\right)$ such that $\left(\nabla u_{\varepsilon}\right)_{\varepsilon>0}$ two-scale converges to $(x, y) \mapsto$ $\nabla_{x} u_{*}(x)+\nabla_{y} u_{1}(x, y)$.

We observe that $u_{1}$ is defined up to the addition of a constant. Similar results could be stated in the framework of the periodic unfolding approach of [20], but since the point of our paper is not to look for optimal regularity condition on $\phi$, we will use the setting of the more standard theory of two-scale convergence. 
5.4. Some compactness properties. The interplay of the Gaussian measure with Lebesgue's measure is essential for getting moments and provides a tight compactness property in the framework of bounded measures. On the other hand, the logarithmic Sobolev inequality prevents concentration. Altogether, this results in a compactness property which can be stated as follows.

Lemma 5.6. The embedding $H^{1}\left(\mathbb{R}^{d}, d \mu_{0}\right) \hookrightarrow L^{2}\left(\mathbb{R}^{d}, d \mu_{0}\right)$ is compact.

Proof. Let $\left(u_{n}\right)_{n \in \mathbb{N}}$ be a sequence of functions in $H^{1}\left(\mathbb{R}^{d}, d \mu_{0}\right)$ which is such that $\left\|u_{n}\right\|_{H^{1}\left(\mathbb{R}^{d}, d \mu_{0}\right)}^{2} \leq 1$ and define $u_{n}:=v_{n} / \sqrt{\mu_{0}}$. By expanding and integrating by parts the square we have

$$
\int_{\mathbb{R}^{d}}\left|\nabla u_{n}\right|^{2} d \mu_{0}=\int_{\mathbb{R}^{d}}\left|\nabla v_{n}\right|^{2} d x+\frac{1}{4} \int_{\mathbb{R}^{d}}|x|^{2}\left|v_{n}\right|^{2} d x-\frac{d}{2} \int_{\mathbb{R}^{d}}\left|v_{n}\right|^{2} d x .
$$

Hence $\int_{\mathbb{R}^{d}}\left|\nabla v_{n}\right|^{2} d x$ and $\int_{\mathbb{R}^{d}}|x|^{2}\left|v_{n}\right|^{2} d x$ are simultaneously uniformly bounded. Using

$\int_{\mathbb{R}^{d}}\left|u_{n}\right|^{2} \log \left|u_{n}\right|^{2} d \mu_{0}=\int_{\mathbb{R}^{d}}\left|v_{n}\right|^{2} \log \left|v_{n}\right|^{2} d x+\log Z_{0} \int_{\mathbb{R}^{d}}\left|v_{n}\right|^{2} d x+\frac{1}{2} \int_{\mathbb{R}^{d}}|x|^{2}\left|v_{n}\right|^{2} d x$

and the logarithmic Sobolev inequality, with $\mathcal{C}_{0}^{(1)}=1 / 2$ :

$$
\int_{\mathbb{R}^{d}}|\nabla u|^{2} d \mu_{0} \geq \mathcal{C}_{0}^{(1)} \int_{\mathbb{R}^{d}}|u|^{2} \log \left(\frac{|u|^{2}}{\int_{\mathbb{R}^{d}}|u|^{2} d \mu_{0}}\right) d \mu_{0} \quad \forall u \in H^{1}\left(\mathbb{R}^{d}, d \mu_{0}\right),
$$

we also know that $\int_{\mathbb{R}^{d}}\left|v_{n}\right|^{2} \log \left|v_{n}\right|^{2} d x$ is also uniformly bounded. By Dunford-Pettis' theorem, $\left(\left|v_{n}\right|^{2}\right)_{n \in \mathbb{N}}$ is weakly compact in $L^{1}\left(\mathbb{R}^{d}, d x\right)$. Denote by $v$ the weak limit in $L^{2}\left(\mathbb{R}^{d}, d x\right)$ of $\left(v_{n}\right)_{n \in \mathbb{N}}$, after extraction of a subsequence if necessary. By Sobolev's embedding, we also know that $v_{n}$ converges almost everywhere to $v$. Hence $\left(\left|v_{n}\right|^{2}\right)_{n \in \mathbb{N}}$ weakly converges in $L^{1}\left(\mathbb{R}^{d}, d x\right)$ to $|v|^{2}$, using:

$$
\begin{aligned}
\lim _{n \rightarrow \infty} \int_{\mathbb{R}^{d}}\left|u_{n}-u\right|^{2} d \mu_{0} & =\lim _{n \rightarrow \infty} \int_{\mathbb{R}^{d}}\left|v_{n}-v\right|^{2} d x \\
& =\lim _{n \rightarrow \infty} \int_{\mathbb{R}^{d}}\left|v_{n}\right|^{2} d x+\int_{\mathbb{R}^{d}}|v|^{2} d x-2 \lim _{n \rightarrow \infty} \int_{\mathbb{R}^{d}} v_{n} v d x \\
& =\lim _{n \rightarrow \infty} \int_{\mathbb{R}^{d}}\left|v_{n}\right|^{2} d x-\int_{\mathbb{R}^{d}}|v|^{2} d x=0 .
\end{aligned}
$$

Notice that much more is known:

$$
\begin{gathered}
\nabla u_{n} \rightarrow \nabla u \quad \text { in } L^{2}\left(\mathbb{R}^{d}, d \mu_{0}\right) \\
\nabla v_{n} \rightarrow \nabla v \quad \text { in } L^{2}\left(\mathbb{R}^{d}, d x\right) \\
v_{n} \rightarrow v \quad \text { in } L^{2}\left(\mathbb{R}^{d}, d x\right) \\
x v_{n} \rightarrow x v \quad \text { in } L^{2}\left(\mathbb{R}^{d}, d x\right) \\
x u_{n} \rightarrow x u \quad \text { in } L^{2}\left(\mathbb{R}^{d}, d \mu_{0}\right)
\end{gathered}
$$

The same results as in Lemma 5.6 also hold under the weaker assumption that $\left(\left|\nabla u_{n}\right|^{2}\right)_{n \in \mathbb{N}}$ and $\left(\left|u_{n}\right|\right)_{n \in \mathbb{N}}$ are uniformly bounded, thanks to Poincaré's inequality, with $\mathcal{C}_{0}^{(2)}=1$ :

$$
\int_{\mathbb{R}^{d}}|\nabla u|^{2} d \mu_{0} \geq \mathcal{C}_{0}^{(2)} \int_{\mathbb{R}^{d}}\left|u-\int_{\mathbb{R}^{d}} u d \mu_{0}\right|^{2} d \mu_{0} \quad \forall u \in H^{1}\left(\mathbb{R}^{d}, d \mu_{0}\right) .
$$


As a consequence of Lemmas 5.2 and 5.6, and of the results of Section 5.3, we obtain the following result.

CoROLlaRY 5.7. Let $\left(\varepsilon_{n}\right)_{n \in \mathbb{N}}$ be a decreasing sequence such that $\lim _{n \rightarrow \infty} \varepsilon_{n}=0$ and consider a sequence $\left(u_{n}\right)_{n \in \mathbb{N}}$ of functions in $H^{1}\left(\mathbb{R}^{d}, d \mu_{\varepsilon_{n}}\right)$ such that $\left(\left|\nabla u_{n}\right|^{2}\right)_{n \in \mathbb{N}}$ and $\left(\left|u_{n}\right|\right)_{n \in \mathbb{N}}$ are uniformly bounded. Then there exists a function $u \in H^{1}\left(\mathbb{R}^{d}, d \mu_{0}\right)$ such that, up to extraction of a subsequence, $u_{n}$ converges to $u$ weakly in $H^{1}\left(\mathbb{R}^{d}, d \mu_{0}\right)$, strongly in $L^{2}\left(\mathbb{R}^{d}, d \mu_{0}\right)$ and $u_{n} e^{-\phi\left(x / \varepsilon_{n}\right)}$ as a function in $L^{2}\left(\mathbb{R}^{d}, d \mu_{0}\right)$ two-scale converges to $u(x) e^{-\phi(y)}$.

5.5. A uniform integrability estimate. Define $\lambda_{\varepsilon}:=1 / \mathcal{C}_{\varepsilon}^{(2)}$.

Lemma 5.8. Assume that $\phi \in L^{\infty}\left(\mathbb{T}^{d}\right)$. There exists a positive constant $K_{\varepsilon}$ such that, for any $u \in H^{1}\left(d \mu_{\varepsilon}\right)$ with $\int_{\mathbb{R}^{d}} u d \mu_{\varepsilon}=0$,

$$
\begin{aligned}
& \int_{\Omega_{1}}|u|^{2} \log |u|^{2} d \mu_{\varepsilon} \\
& \quad \leq \frac{1}{e}+K_{\varepsilon} \int_{\mathbb{R}^{d}}|\nabla u|^{2} d \mu_{\varepsilon}+\left(1+\lambda_{\varepsilon} \int_{\mathbb{R}^{d}}|\nabla u|^{2} d \mu_{\varepsilon}\right) \log \left(1+\lambda_{\varepsilon} \int_{\mathbb{R}^{d}}|\nabla u|^{2} d \mu_{\varepsilon}\right)
\end{aligned}
$$

where $\Omega_{1}:=\left\{x \in \mathbb{R}^{d}: u(x) \geq 1\right.$ a.e. $\}$.

Proof. We recall that by definition of $\mathcal{C}_{\varepsilon}^{(2)}$, using $\int_{\mathbb{R}^{d}} u d \mu_{\varepsilon}=0$, we have

$$
\mathcal{C}_{\varepsilon}^{(2)} \int_{\mathbb{R}^{d}}|u|^{2} d \mu_{\varepsilon} \leq \int_{\mathbb{R}^{d}}|\nabla u|^{2} d \mu_{\varepsilon} .
$$

Using the monotonicity of $t \mapsto t \log t$ on $(1, \infty)$, it follows that

$$
\begin{aligned}
\left(\int_{\mathbb{R}^{d}}|u|^{2} d \mu_{\varepsilon}\right) \log \left(\int_{\mathbb{R}^{d}}|u|^{2} d \mu_{\varepsilon}\right) & \leq\left(1+\int_{\mathbb{R}^{d}}|u|^{2} d \mu_{\varepsilon}\right) \log \left(1+\int_{\mathbb{R}^{d}}|u|^{2} d \mu_{\varepsilon}\right) \\
& \leq\left(1+\lambda_{\varepsilon} \int_{\mathbb{R}^{d}}|\nabla u|^{2} d \mu_{\varepsilon}\right) \log \left(1+\lambda_{\varepsilon} \int_{\mathbb{R}^{d}}|\nabla u|^{2} d \mu_{\varepsilon}\right) .
\end{aligned}
$$

By definition of $\mathcal{C}_{\varepsilon}^{(1)}$, we obtain

$$
\begin{array}{r}
\int_{\mathbb{R}^{d}}|u|^{2} \log |u|^{2} d \mu_{\varepsilon} \leq\left(1+\lambda_{\varepsilon} \int_{\mathbb{R}^{d}}|\nabla u|^{2} d \mu_{\varepsilon}\right) \log \left(1+\lambda_{\varepsilon} \int_{\mathbb{R}^{d}}|\nabla u|^{2} d \mu_{\varepsilon}\right) \\
+\frac{1}{\mathcal{C}_{\varepsilon}^{(1)}} \int_{\mathbb{R}^{d}}|\nabla u|^{2} d \mu_{\varepsilon} .
\end{array}
$$

Now, by (5.3), we have

$$
\int_{\mathbb{R}^{d}}|x|^{2}|u|^{2} d \mu_{0} \leq 4 \int_{\mathbb{R}^{d}}|\nabla u|^{2} d \mu_{0}+2 d \int_{\mathbb{R}^{d}}|u|^{2} d \mu_{0} .
$$

We can then write

$$
e^{-\|\phi\|_{L^{\infty}\left(\mathbb{T}^{d}\right)}} \int_{\mathbb{R}^{d}}|x|^{2}|u|^{2} d \mu_{\varepsilon} \leq 4 \int_{\mathbb{R}^{d}}|\nabla u|^{2} d \mu_{\varepsilon}+2 d \int_{\mathbb{R}^{d}}|u|^{2} d \mu_{\varepsilon},
$$

which, combined with (5.4), amounts to

$$
\int_{\mathbb{R}^{d}}|x|^{2}|u|^{2} d \mu_{\varepsilon} \leq 2 e^{\|\phi\|_{L^{\infty}\left(\mathbb{T}^{d}\right)}}\left(2+d \lambda_{\varepsilon}\right) \int_{\mathbb{R}^{d}}|\nabla u|^{2} d \mu .
$$


The next step is an adaptation of the so-called Carleman estimate. Let $Z_{\varepsilon}:=$ $\int_{\Omega_{1}^{c}} e^{-|x|^{2}} d \mu_{\varepsilon}$. On $\Omega_{1}^{c}$, we have

$$
\int_{\Omega_{1}^{c}}|u|^{2}\left(\log |u|^{2}+|x|^{2}\right) d \mu_{\varepsilon}=\int_{\Omega_{1}^{c}}|u|^{2} \log \left(\frac{|u|^{2}}{Z_{\varepsilon}^{-1} e^{-|x|^{2}}}\right) d \mu_{\varepsilon}-\log Z_{\varepsilon} \int_{\Omega_{1}^{c}}|u|^{2} d \mu_{\varepsilon}
$$

which is bounded from below, using Jensen's inequality and the convexity of $t \mapsto$ $t \log t$, by

$$
\begin{aligned}
\int_{\Omega_{1}^{c}}\left(\frac{|u|^{2}}{Z_{\varepsilon}^{-1} e^{-|x|^{2}}}\right) \log \left(\frac{|u|^{2}}{Z_{\varepsilon}^{-1} e^{-|x|^{2}}}\right) Z_{\varepsilon}^{-1} e^{-|x|^{2}} d \mu_{\varepsilon} & \\
& \geq\left(\int_{\Omega_{1}^{c}}|u|^{2} d \mu_{\varepsilon}\right) \log \left(\int_{\Omega_{1}^{c}}|u|^{2} d \mu_{\varepsilon}\right) .
\end{aligned}
$$

As a consequence, using $\sup _{t>0}-t \log t=1 / e$, we obtain

$$
\begin{aligned}
&\left.\left|\int_{\Omega_{1}^{c}}\right| u\right|^{2} \log |u|^{2} d \mu_{\varepsilon} \mid=-\int_{\Omega_{1}^{c}}|u|^{2} \log |u|^{2} d \mu_{\varepsilon} \\
& \leq \log Z_{\varepsilon} \int_{\Omega_{1}^{c}}|u|^{2} d \mu_{\varepsilon}+\frac{1}{e}+\int_{\Omega_{1}^{c}}|x|^{2}|u|^{2} d \mu_{\varepsilon}, \\
&\left.\left.\left|\int_{\Omega_{1}^{c}}\right| u\right|^{2} \log |u|^{2} d \mu_{\varepsilon}\left|\leq \log \left(\int_{\mathbb{R}^{d}} e^{-|x|^{2}} d \mu_{\varepsilon}\right) \int_{\mathbb{R}^{d}}\right| u\right|^{2} d \mu_{\varepsilon}+\frac{1}{e}+\int_{\mathbb{R}^{d}}|x|^{2}|u|^{2} d \mu_{\varepsilon} .
\end{aligned}
$$

We conclude by writing

$$
\int_{\Omega_{1}}|u|^{2} \log |u|^{2} d \mu_{\varepsilon}=\int_{\mathbb{R}^{d}}|u|^{2} \log |u|^{2} d \mu_{\varepsilon}+\left.\left|\int_{\Omega_{1}^{c}}\right| u\right|^{2} \log |u|^{2} d \mu_{\varepsilon} \mid
$$

and using (5.4)-(5.6).

Let $\Omega_{A}:=\left\{x \in \mathbb{R}^{d}: u(x) \geq A\right.$ a.e. $\}$ for any $A \geq 1$. A straightforward consequence of Lemma 5.8 is the following uniform integrability property.

Corollary 5.9. Assume that $\phi \in L^{\infty}\left(\mathbb{T}^{d}\right)$. With the above notations, for any $B>0$, there exists $a C_{\varepsilon}(B)>0$ which depends only on $\varepsilon$ and $B$, such that, for any $u \in H^{1}\left(d \mu_{\varepsilon}\right)$ such that $\int_{\mathbb{R}^{d}} u d \mu_{\varepsilon}=0$, if $\int_{\mathbb{R}^{d}}|\nabla u|^{2} d \mu_{\varepsilon} \leq B$, then

$$
\int_{\Omega_{A}}|u|^{2} d \mu_{\varepsilon} \leq \frac{C_{\varepsilon}(B)}{\log \left(A^{2}\right)} \quad \forall A \geq 1
$$

5.6. Interpolation between the Poincaré inequality and the logarithmic Sobolev inequality. We first recall a standard comparison result for the best constants.

Lemma 5.10. For any $p \in[1,2], \mathcal{C}_{\varepsilon}^{(p)} \leq \frac{p}{2} \mathcal{C}_{\varepsilon}^{(2)}$.

Proof. Assume that $p \in(1,2)$. By definition of $\mathcal{C}_{\varepsilon}^{(p)}$, for any $u \in H^{1}\left(d \mu_{\varepsilon}\right)$,

$$
\mathcal{C}_{\varepsilon}^{(p)} \frac{\int_{\mathbb{R}^{d}}|u|^{2} d \mu_{\varepsilon}-\left(\int_{\mathbb{R}^{d}}|u|^{2 / p} d \mu_{\varepsilon}\right)^{p}}{p-1} \leq \int_{\mathbb{R}^{d}}|\nabla u|^{2} d \mu_{\varepsilon},
$$


Assume that $u=1+\eta v$ and consider the limit $\eta \rightarrow 0$. A Taylor Expansion at order two in $\eta$ shows that

$$
\frac{2}{p} \mathcal{C}_{\varepsilon}^{(p)} \eta^{2}\left[\int_{\mathbb{R}^{d}}|v|^{2} d \mu_{\varepsilon}-\left(\int_{\mathbb{R}^{d}}|v| d \mu_{\varepsilon}\right)^{2}\right](1+o(\eta)) \leq \eta^{2} \int_{\mathbb{R}^{d}}|\nabla v|^{2} d \mu_{\varepsilon} \quad \text { as } \quad \eta \rightarrow 0,
$$

which proves the estimate. A similar computation also holds in the case $p=1$.

The next result is taken from [4] (also see [34] for an earlier partial result).

TheOREm 5.11. [4] For any $p \in[1,2]$, the following estimate holds

$$
\mathcal{C}_{\varepsilon}^{(2)} \leq \frac{1}{p-1}\left[1-\left(\frac{2-p}{p}\right)^{\alpha}\right] \mathcal{C}_{\varepsilon}^{(p)} \quad \text { with } \quad \alpha:=\frac{\mathcal{C}_{\varepsilon}^{(2)}}{2 \mathcal{C}_{\varepsilon}^{(1)}}
$$

As a straightforward consequence of Lemma 5.10 and Theorem 5.11, we get the following result.

Corollary 5.12. [4] If $\mathcal{C}_{\varepsilon}^{(1)}=\frac{1}{2} \mathcal{C}_{\varepsilon}^{(2)}$, then $\mathcal{C}_{\varepsilon}^{(p)}=\frac{p}{2} \mathcal{C}_{\varepsilon}^{(2)}$ for any $p \in[1,2]$.

As observed by Latała and Oleskiewicz in [34], for a given $u \in H^{1}\left(d \mu_{\varepsilon}\right)$, the function $g(p):=p \log \left(\int_{\mathbb{R}^{d}}|u|^{2 / p} d \mu_{\varepsilon}\right)$ is such that

$$
\begin{aligned}
\frac{p^{3}}{4}\left(\int_{\mathbb{R}^{d}}|u|^{2 / p} d \mu_{\varepsilon}\right)^{2} g^{\prime \prime}(p) & \\
& =\int_{\mathbb{R}^{d}}|u|^{2 / p}(\log |u|)^{2} d \mu_{\varepsilon} \int_{\mathbb{R}^{d}}|u|^{2 / p} d \mu_{\varepsilon}-\left(\int_{\mathbb{R}^{d}}|u|^{2 / p} \log |u| d \mu_{\varepsilon}\right)^{2}
\end{aligned}
$$

is nonnegative by the Cauchy-Schwarz inequality, so that $g$ is convex. Hence $f(p)=$ $e^{g(p)}$ is also convex because $f^{\prime \prime}(p)=\left(g^{\prime \prime}(p)+g^{\prime}(p)^{2}\right) f(p)$. This proves that, for any $p \in(1,2]$,

$$
\begin{aligned}
-\frac{f(p)-f(1)}{p-1} & =\frac{\int_{\mathbb{R}^{d}}|u|^{2} d \mu_{\varepsilon}-\left(\int_{\mathbb{R}^{d}}|u|^{2 / p}\right)^{p} d \mu_{\varepsilon}}{p-1} \\
& \leq \int_{\mathbb{R}^{d}}|u|^{2} \log \left(\frac{|u|^{2}}{\int_{\mathbb{R}^{d}}|u|^{2} d \mu_{\varepsilon}}\right) d \mu_{\varepsilon}=-f^{\prime}(1) .
\end{aligned}
$$

There is however no a priori reason to expect that $f^{\prime \prime}(1)$ should be finite for an arbitrary $u \in H^{1}\left(d \mu_{\varepsilon}\right)$.

\section{Proof of Theorem 4.1.}

6.1. The Poincaré inequality. We start with the case $p=2$. As a consequence of Corollary 5.7, for any $\varepsilon>0$, there exists a non-trivial minimizer $u_{\varepsilon}$ to $\mathcal{C}_{\varepsilon}^{(2)}$ such that $\int_{\mathbb{R}^{d}} u_{\varepsilon} d \mu_{\varepsilon}=0, \int_{\mathbb{R}^{d}}\left|u_{\varepsilon}\right|^{2} d \mu_{\varepsilon}=1$ and

$$
-\nabla \cdot\left(e^{-\frac{1}{2}|x|^{2}-\phi(x / \varepsilon)} \nabla u_{\varepsilon}(x)\right)=\mathcal{C}_{\varepsilon}^{(2)} u_{\varepsilon}(x) e^{-\frac{1}{2}|x|^{2}-\phi(x / \varepsilon)} .
$$

Let $\varphi \in \mathcal{D}\left(\mathbb{R}^{d}\right)$ and $\varphi_{1} \in \mathcal{D}\left(\mathbb{R}^{d}, C^{\infty}\left(\mathbb{T}^{d}\right)\right)$. We test $(6.1)$ by $\varphi+\varepsilon \varphi_{1}(\cdot, \cdot / \varepsilon)$. Integrating by parts this yields

$$
\begin{aligned}
\int_{\mathbb{R}^{d}} \nabla_{x} u_{\varepsilon}\left[\nabla_{x} \varphi(x)+\varepsilon \nabla_{x} \varphi_{1}\left(x, \frac{x}{\varepsilon}\right)+\nabla_{y} \varphi_{1}\left(x, \frac{x}{\varepsilon}\right)\right] d \mu_{\varepsilon} & \\
& =\mathcal{C}_{\varepsilon}^{(2)} \int_{\mathbb{R}^{d}} u_{\varepsilon}\left[\varphi(x)+\varepsilon \varphi_{1}\left(x, \frac{x}{\varepsilon}\right)\right] d \mu_{\varepsilon}
\end{aligned}
$$


As $\varepsilon \rightarrow 0_{+}$, up to the extraction of a subsequence, the sequence $\left(u_{\varepsilon}\right)_{\varepsilon>0}$, not relabelled, weakly converges in $H^{1}\left(\mathbb{R}^{d}, d \mu_{0}\right)$ to some function $u_{*}$, and according to Proposition 5.5, there exists a function $u_{1} \in L^{2}\left(\mathbb{R}^{d}, H^{1}\left(\mathbb{T}^{d}\right)\right)$ such that $\left(\nabla u_{\varepsilon}\right)_{\varepsilon>0}$ two-scale converges to $(x, y) \mapsto \nabla_{x} u_{*}(x)+\nabla_{y} u_{1}(x, y)$. Let $\mathcal{K}_{0}^{(2)}:=\lim _{\varepsilon \rightarrow 0_{+}} \mathcal{C}_{\varepsilon}^{(2)}$. Taking the limit $\varepsilon \rightarrow 0_{+}$, we obtain a two-scale homogenized equation:

$$
\begin{aligned}
\iint_{\mathbb{R}^{d} \times \mathbb{T}^{d}}\left[\nabla_{x} u_{*}(x)+\nabla_{y} u_{1}(x, y)\right] & {\left[\nabla_{x} \varphi(x)+\nabla_{y} \varphi_{1}(x, y)\right] e^{-\frac{1}{2}|x|^{2}-\phi(y)} d x d y } \\
& =\mathcal{K}_{0}^{(2)} \iint_{\mathbb{R}^{d} \times \mathbb{T}^{d}} u_{*}(x) \varphi(x) e^{-\frac{1}{2}|x|^{2}-\phi(y)} d x d y .
\end{aligned}
$$

An evaluation with $\varphi=0$ shows that $u_{1}$ is given as a solution of

$$
\nabla_{y} \cdot\left[e^{-\phi(y)}\left(\nabla_{y} u_{1}(x, y)+\nabla_{x} u_{*}(x)\right)\right]=0,
$$

Exactly as in the introduction, this amounts to write that

$$
u_{1}(x, y)=\nabla_{x} u_{*}(x) \cdot w(y)
$$

where $w=\left(w_{j}\right)_{j=1}^{d}$ is the solution of the cell equation (3.5). Using (3.6), we find that

$$
\nabla_{y} u_{1}(x, y)=\left[\frac{e^{\phi}}{\int_{\mathbb{T}^{d}} e^{\phi(y)} d y}-1\right] \nabla_{x} u_{*}(x) .
$$

By testing (6.2) with $\varphi=u_{*}$ (up to an appropriate regularization procedure if necessary) and $\varphi_{1}=0$, and using (3.2), we get

$$
\int_{\mathbb{R}^{d}} \frac{\left|\nabla_{x} u_{*}\right|^{2}}{\int_{\mathbb{T}^{d}} e^{\phi(y)} d y} d \mu_{0}=\mathcal{K}_{0}^{(2)} \int_{\mathbb{R}^{d}}\left|u_{*}\right|^{2} d \mu_{0}
$$

We can also observe that

$$
\int_{\mathbb{R}^{d}} u_{*} d \mu_{0}=\lim _{\varepsilon \rightarrow 0_{+}} \int_{\mathbb{R}^{d}} u_{\varepsilon} d \mu_{\varepsilon}=0 .
$$

Altogether this proves that

$$
\mathcal{K}_{0}^{(2)} \geq \frac{\mathcal{C}_{0}^{(2)}}{\int_{\mathbb{T}^{d}} e^{\phi(y)} d y}=\mathrm{K} \mathcal{C}_{0}^{(2)}
$$

On the other hand, it is not a priori granted that $u_{*}$ is optimal for $\mathcal{C}_{0}^{(2)}$. However, if $w$ is the solution of (3.5) and if we use

$$
\tilde{u}_{\varepsilon}(x):=u_{\mathrm{e}}(x)+\varepsilon \nabla_{x} u_{\mathrm{e}}(x) w\left(\frac{x}{\varepsilon}\right)
$$

where $u_{\mathrm{e}}(x)=x \cdot \mathrm{e}$ has already been defined in Section 5.1 and is optimal for $\mathcal{C}_{0}^{(2)}$, we find by two-scale convergence that

$$
\mathcal{K}_{0}^{(2)} \leq \lim _{\varepsilon \rightarrow 0_{+}} \frac{\int_{\mathbb{R}^{d}}\left|\nabla \tilde{u}_{\varepsilon}\right|^{2} d \mu_{\varepsilon}}{\int_{\mathbb{R}^{d}}\left|\tilde{u}_{\varepsilon}\right|^{2} d \mu_{\varepsilon}-\left(\int_{\mathbb{R}^{d}} \tilde{u}_{\varepsilon} d \mu_{\varepsilon}\right)^{2}}=\mathrm{KC}_{0}^{(2)} .
$$

This completes the proof of Theorem 4.1 in case $p=2$. 
6.2. The generalized Poincaré inequality. For $p \in[1,2)$, deciding whether $\mathcal{C}_{\varepsilon}^{(p)}$ is achieved or not by some non trivial function $u \in H^{1}\left(\mathbb{R}^{d}, d \mu_{\varepsilon}\right)$ is a difficult question. For $\varepsilon=0$, in the case $p=1$, E. Carlen and M. Loss in [16] proved that $\mathcal{C}_{0}^{(2)}$ is not achieved (equality in the logarithmic Sobolev inequality with Gaussian weight holds only for constants, which are explicitly excluded when taking the infimum). Here we establish a much simpler result which is sufficient to conclude in the proof of Theorem 4.1. The following result is inspired by a result in [42] for the logarithmic Sobolev inequality.

Proposition 6.1. Let $\phi$ be a continuous function on $\mathbb{T}^{d}$ and take $p \in(1,2)$, $\varepsilon>0$. Then, with the above notations, either

$$
\mathcal{C}_{\varepsilon}^{(p)} \leq \frac{p}{2} \mathcal{C}_{\varepsilon}^{(2)}
$$

is achieved by some non trivial function, or

$$
\mathcal{C}_{\varepsilon}^{(p)}=\frac{p}{2} \mathcal{C}_{\varepsilon}^{(2)}
$$

is not achieved by any non trivial function.

Proof. Let $u_{\varepsilon}$ be an optimal function for $\mathcal{C}_{\varepsilon}^{(2)}$. An elementary computation shows that

$$
\mathcal{C}_{\varepsilon}^{(p)} \leq \lim _{n \rightarrow \infty} \frac{(p-1) \int_{\mathbb{R}^{d}}\left|\nabla u_{\varepsilon}^{n}\right|^{2} d \mu_{\varepsilon}}{\int_{\mathbb{R}^{d}}\left|u_{\varepsilon}^{n}\right|^{2} d \mu_{\varepsilon}-\left(\int_{\mathbb{R}^{d}}\left|u_{\varepsilon}^{n}\right|^{2 / p} d \mu_{\varepsilon}\right)^{p}}=\frac{p}{2} \mathcal{C}_{\varepsilon}^{(2)}
$$

where

$$
u_{\varepsilon}^{n}:=1+\frac{1}{n} u_{\varepsilon} .
$$

Consider now a minimizing sequence $\left(u_{n}\right)_{n \in \mathbb{N}}$,

$$
\lim _{n \rightarrow \infty} \frac{(p-1) \int_{\mathbb{R}^{d}}\left|\nabla u_{n}\right|^{2} d \mu_{\varepsilon}}{\int_{\mathbb{R}^{d}}\left|u_{n}\right|^{2} d \mu_{\varepsilon}-\left(\int_{\mathbb{R}^{d}}\left|u_{n}\right|^{2 / p} d \mu_{\varepsilon}\right)^{p}}=\mathcal{C}_{\varepsilon}^{(p)},
$$

for which we additionally assume that $\int_{\mathbb{R}^{d}}\left|\nabla u_{n}\right|^{2} d \mu_{\varepsilon} \neq 0$ for any $n \in \mathbb{N}$ and, using the homogeneity, $\int_{\mathbb{R}^{d}}\left|u_{n}\right|^{2} d \mu_{\varepsilon}=1$. By Hölder's inequality,

$$
\left(\int_{\mathbb{R}^{d}}\left|u_{n}\right|^{2 / p} d \mu_{\varepsilon}\right)^{p} \leq \int_{\mathbb{R}^{d}}\left|u_{n}\right|^{2} d \mu_{\varepsilon},
$$

so that $\delta_{n}:=\int_{\mathbb{R}^{d}}\left|u_{n}\right|^{2} d \mu_{\varepsilon}-\left(\int_{\mathbb{R}^{d}}\left|u_{n}\right|^{2 / p} d \mu_{\varepsilon}\right)^{p} \leq 1$ for any $n \in \mathbb{N}$. Let $\delta=$ $\liminf \operatorname{in}_{n \rightarrow \infty} \delta_{n}$.

If $\delta>0$, then $\left(u_{n}\right)_{n \in \mathbb{N}}$ strongly converges to some function $u$ in $L^{1} \cap L^{2}\left(\mathbb{R}^{d}, d \mu_{\varepsilon}\right)$, up to the extraction of a subsequence, and by lower semi-continuity, $\int_{\mathbb{R}^{d}}|\nabla u|^{2} d \mu_{\varepsilon} \leq$ $\liminf _{n \rightarrow \infty} \int_{\mathbb{R}^{d}}\left|\nabla u_{n}\right|^{2} d \mu_{\varepsilon}$. Moreover, since $\int_{\mathbb{R}^{d}}|u|^{2} d \mu_{\varepsilon}-\left(\int_{\mathbb{R}^{d}}|u|^{2 / p} d \mu_{\varepsilon}\right)^{p}=\delta>0$, $u$ is not constant and it is therefore a minimizer for $\mathcal{C}_{\varepsilon}^{(p)}$.

If $\delta=0$, then, up to the extraction of a subsequence, $\eta_{n}:=\left(\int_{\mathbb{R}^{d}}\left|\nabla u_{n}\right|^{2} d \mu_{\varepsilon}\right)^{1 / 2}$ also converges to 0 and almost everywhere, $\lim _{n \rightarrow \infty} u_{n}(x)=\lim _{n \rightarrow \infty} \bar{u}_{n}=1$ where

$$
\bar{u}_{n}:=\int_{\mathbb{R}^{d}} u_{n} d \mu_{\varepsilon}
$$


The sequence $\left(v_{n}\right)_{n \in \mathbb{N}^{*}}$ with $v_{n}:=\frac{u_{n}-\bar{u}_{n}}{\eta_{n} \bar{u}_{n}}$ is such that, for any $n \in \mathbb{N}$,

$$
\begin{gathered}
\int_{\mathbb{R}^{d}}\left|\nabla v_{n}\right|^{2} d \mu_{\varepsilon}=\frac{1}{\bar{u}_{n}^{2}}, \quad \int_{\mathbb{R}^{d}} v_{n} d \mu_{\varepsilon}=0, \\
\frac{\delta_{n}}{\bar{u}_{n}^{2} \eta_{n}^{2}}=\int_{\mathbb{R}^{d}}\left|1+\eta_{n} v_{n}\right|^{2} d \mu_{\varepsilon}-\left(\int_{\mathbb{R}^{d}}\left|1+\eta_{n} v_{n}\right|^{2 / p} d \mu_{\varepsilon}\right)^{p} .
\end{gathered}
$$

For any $p \in(1,2)$, there exists a positive constant $c_{p}$ such that

$$
|| 1+\left.x\right|^{\frac{2}{p}}-1-\frac{2}{p} x-\frac{2-p}{p^{2}} x^{2} \mid \leq c_{p}\left(1+x^{2}\right) \quad \forall x \in \mathbb{R} .
$$

Hence, for $A>1$, large,

$$
\begin{aligned}
\frac{1}{\eta_{n}^{2}}\left|\int_{\mathbb{R}^{d}}\left(\left|1+\eta_{n} v_{n}\right|^{\frac{2}{p}}-1-\frac{2}{p} \eta_{n} v_{n}-\frac{2-p}{p^{2}} \eta_{n}^{2} v_{n}^{2}\right) d \mu_{\varepsilon}\right| & \leq \int_{\left|v_{n}\right| \leq A} f_{n}^{A} v_{n}^{2} d \mu_{\varepsilon} \\
& +c_{p} \int_{\left|v_{n}\right|>A}\left(1+v_{n}^{2}\right) d \mu_{\varepsilon}
\end{aligned}
$$

where $\left(f_{n}^{A}\right)_{n \in \mathbb{N}}$ is a sequence of bounded functions, with a bound depending on $A$, which converges almost everywhere to 0 . The term $\int_{\left|v_{n}\right| \leq A} f_{n}^{A} v_{n}^{2} d \mu_{\varepsilon}$ converges to 0 by Lebesgue's theorem of dominated convergence. On the other hand, by Corollary 5.9 , we get

$$
\int_{\left|v_{n}\right|>A}\left(1+v_{n}^{2}\right) d \mu_{\varepsilon} \leq \frac{1}{A^{2}}+\frac{C_{\varepsilon}\left(1 / \bar{u}_{n}^{2}\right)}{\log A^{2}} .
$$

Summarizing, we have found that

$$
\begin{gathered}
\int_{\mathbb{R}^{d}}\left|1+\eta_{n} v_{n}\right|^{\frac{2}{p}} d \mu_{\varepsilon}=1+\frac{2}{p} \eta_{n} \underbrace{\int_{\mathbb{R}^{d}} v_{n} d \mu_{\varepsilon}}_{=0}+\frac{2-p}{p^{2}} \eta_{n}^{2} \int_{\mathbb{R}^{d}} v_{n}^{2} d \mu_{\varepsilon}+o\left(\eta_{n}^{2}\right), \\
\delta_{n}=\frac{2(p-1)}{p} \int_{\mathbb{R}^{d}} v_{n}^{2} d \mu_{\varepsilon} \eta_{n}^{2}+o\left(\eta_{n}^{2}\right)+O\left(\frac{1}{A^{2}}+\frac{1}{\log A}\right) .
\end{gathered}
$$

Since $\int_{\mathbb{R}^{d}}\left|\nabla v_{n}\right|^{2} d \mu_{\varepsilon}=1, A$ can be chosen arbitrarily large and

$$
\mathcal{C}_{\varepsilon}^{(p)}=\frac{p}{2} \lim _{n \rightarrow \infty} \frac{\int_{\mathbb{R}^{d}}\left|\nabla v_{n}\right|^{2} d \mu_{\varepsilon} \eta_{n}^{2}}{\int_{\mathbb{R}^{d}} v_{n}^{2} d \mu_{\varepsilon} \eta_{n}^{2}+o\left(\eta_{n}^{2}\right)},
$$

it turns out that $\liminf _{n \rightarrow \infty} \int_{\mathbb{R}^{d}} v_{n}^{2} d \mu_{\varepsilon}>0$, so that

$$
\mathcal{C}_{\varepsilon}^{(p)}=\frac{p}{2} \lim _{n \rightarrow \infty} \frac{\int_{\mathbb{R}^{d}}\left|\nabla v_{n}\right|^{2} d \mu_{\varepsilon}}{\int_{\mathbb{R}^{d}} v_{n}^{2} d \mu_{\varepsilon}} \geq \frac{p}{2} \mathcal{C}_{\varepsilon}^{(2)}
$$

thus completing the proof.

Proof of Theorem 4.1 if $p \in(1,2)$. Let $\left(\varepsilon_{n}\right)_{n \in \mathbb{N}}$ be a decreasing sequence such that $\lim _{n \rightarrow \infty} \varepsilon_{n}=0$. Up to the extraction of a subsequence, if $\mathcal{C}_{\varepsilon_{n}}^{(p)}=\frac{p}{2} \mathcal{C}_{\varepsilon_{n}}^{(2)}$ for any $n \in \mathbb{N}$, then $\lim _{n \rightarrow \infty} \mathcal{C}_{\varepsilon_{n}}^{(p)}=\mathrm{KC}_{0}^{(p)}$. Otherwise, $\mathcal{C}_{\varepsilon_{n}}^{(p)}<\frac{p}{2} \mathcal{C}_{\varepsilon_{n}}^{(2)}$ for $n$ large enough. According to Proposition $6.1, \mathcal{C}_{\varepsilon_{n}}^{(p)}$ admits a minimizer. The same analysis as in the case $p=2$ can be done, thus proving again that $\lim _{n \rightarrow \infty} \mathcal{C}_{\varepsilon_{n}}^{(p)}=\mathrm{KC}_{0}^{(p)}$. 
6.3. The logarithmic Sobolev inequality. The case $p=1$ is not completely understood. The lower bound on $\lim _{\varepsilon \rightarrow 0_{+}} \mathcal{C}_{\varepsilon}^{(1)}$ follows from Corollary 5.3. The upper bound is a consequence of Corollary 5.12. One can also get a direct proof as in the proof of Proposition 6.1, by considering $u_{\varepsilon}^{n}:=1+\frac{1}{n} u_{\varepsilon}$ where $u_{\varepsilon}$ is an optimal function for $\mathcal{C}_{\varepsilon}^{(2)}$.

7. Rates of convergence. The rate of convergence of the solution of (1.2) corresponding to $u^{\varepsilon}(t, x) \underset{t \rightarrow \infty}{\stackrel{L^{1} \cap L^{2}}{\longrightarrow}} u_{\infty}^{\varepsilon}(x)$ in Diagram (3.9) follows from Theorem 4.1. Notice that $\left\|u^{\varepsilon}-u_{\infty}^{\varepsilon}\right\|_{L^{p}\left(\mathbb{R}^{d},\left(u_{\infty}^{\varepsilon}\right)^{1-p} d x\right)} \leq e^{\|\phi\|_{L^{\infty}\left(\mathbb{T}^{d}\right)}}\left(u_{\infty}^{0}(0)\right)^{1-p}\left\|u^{\varepsilon}-u_{\infty}^{\varepsilon}\right\|_{L^{p}\left(\mathbb{R}^{d}, d x\right)}$.

Corollary 7.1. Assume that $\phi$ is a $C^{2}$ function on $\mathbb{T}^{d}$. With the notations of the introduction, if $u$ is a smooth solution of (1.2), then there exists a constant $\mathrm{A}=\mathrm{A}\left[u_{0}\right]$ such that

$$
\left\|u^{\varepsilon}-u_{\infty}^{\varepsilon}\right\|_{L^{p}\left(\mathbb{R}^{d},\left(u_{\infty}^{\varepsilon}\right)^{1-p} d x\right)}^{2} \leq \mathrm{A} e^{-4 \mathcal{C}_{\varepsilon}^{(p)} t / p} \quad \forall t>0
$$

for any $p \in(1,2]$, where $\lim _{\varepsilon \rightarrow 0_{+}} 4 \mathcal{C}_{\varepsilon}^{(p)} / p=2 \mathrm{~K}<2$, and $\lim _{\varepsilon \rightarrow 0_{+}} 4 \mathcal{C}_{\varepsilon}^{(1)} \leq 2 \mathrm{~K}<2$.

Hence, it is not only that the average profile converges to $v^{(0)}$ which solves (3.8) with a diffusion coefficient $\mathrm{K}$, but also the rate of convergence which is modified by a factor K.

Proof. By Theorem 4.1 and Inequality 3.1,

$$
\mathrm{E}_{\varepsilon}^{(p)}\left[u^{\varepsilon}(t, \cdot)\right] \leq \mathrm{E}_{\varepsilon}^{(p)}\left[u_{0}\right] e^{-4 \mathcal{C}_{\varepsilon}^{(p)} t / p} \quad \forall t>0 .
$$

If $p=2$, the result is already proven. If $p=1$, it follows from the Csiszár-Kullback inequality, see, e.g., [44]. Otherwise, according to the generalized Csiszár-Kullback inequality (see for instance [9], and replace Lebesgue's measure by $d \mu_{\varepsilon}$ ), we have

$$
\left(\int_{\mathbb{R}^{d}}|v-1|^{p} d \mu_{\varepsilon}\right)^{\frac{2}{p}} \leq \frac{2^{\frac{2}{p}}}{p}\left(\int_{\mathbb{R}^{d}}|v|^{p} d \mu_{\varepsilon}\right)^{\frac{2-p}{p}} \int_{\mathbb{R}^{d}} \frac{v^{p}-1-p(v-1)}{p-1} d \mu_{\varepsilon},
$$

which, applied with $v=u^{\varepsilon} / u_{\infty}^{\varepsilon}$, gives the result.

Appendix: an estimate based on Hermite functions. In this appendix, we give a sufficient condition for (2.16) to hold. By constructing a super-solution, we prove that there is a class of solutions $u$ of (2.6) which satisfy $\int_{\mathbb{R}}|x|^{4} u(t, x) d x<\infty$ for any $t>0$. We conjecture that such a property holds for a much larger class of solutions.

Let $\tilde{u}(t, x)=\tilde{\mathrm{U}}(t, x ; z):=\tilde{\mathrm{U}}_{0}+R^{-1} \tilde{\mathrm{U}}_{1}+R^{-2} \tilde{\mathrm{U}}_{2}$, with $z=e^{t} x-\frac{1}{2}\left(e^{2 t}-1\right) A(\omega)$ and $\tilde{U}_{0}:=g_{\omega} \tilde{h}, \tilde{U}_{1}:=g_{\omega}^{(1)} \tilde{h}_{x}, \mathrm{U}_{1}:=g_{\omega}^{(2)} \tilde{h}_{x x}$, and where $\tilde{h}$ is the solution of

$$
\mathrm{L}_{2} \tilde{h}=-e^{-t} \eta, \quad \tilde{h}(t=0, \cdot)=\eta .
$$

Here we choose $\eta \in \mathcal{S}(\mathbb{R})$ to be a smooth even function such that $\eta(x) \geq 1 / e$ if $|x| \leq 1$ and $\eta(x)=\exp (-|x|)$ if $|x| \geq 1$. Tedious computations that we shall omit here show that

$$
\mathbf{L} \tilde{\mathbf{U}} \leq-\mathrm{c} e^{-t} \eta+e^{-t}\left[\mathrm{c}_{0}\left|\tilde{h}_{x t}\right|+\sum_{k=1}^{4} \mathrm{c}_{k}\left|\frac{d^{k} \tilde{h}}{d x^{k}}\right|\right]
$$


for some positive constants c $, c_{k}, k=0,1, \ldots 4$. We notice that the function $\tilde{h}$ and its derivatives decay like $e^{-t}$. As a consequence, there exists $T>0$, depending on $\mathrm{c}$, $\mathrm{c}_{k}, k=0,1, \ldots 4$, such that $\frac{1}{2}\left(e^{2 T}-1\right) A(\omega)$ is integer,

$$
\mathrm{L} \tilde{\mathrm{U}} \leq 0 \quad \forall t \geq T
$$

and

$$
\tilde{u}(T, x)=: B(x)>0 \quad \forall x \in \mathbb{R} .
$$

By undoing the change of variables and using the invariance by translation of (1.1), we obtain the following estimate.

Proposition 7.2. If $\psi$ is $C^{2}$, periodic and if $u$ is a solution of (2.6) with a compactly supported initial data $f_{0}$ such that, with the above notations, for some $K>0$,

$$
f_{0} \leq K B(x) \quad \forall x \in \mathbb{R},
$$

then, $u(t, \cdot) \leq K \tilde{u}(t+T, \cdot)$ and as consequence, $u$ satisfies $(2.16)$.

Proof. By decomposing $\tilde{h}$ on Hermite functions $h_{k}(x)=w_{k}(y) e^{-|y|^{2} / 4}, k \in \mathbb{N}$, with $y=x / \sqrt{\kappa_{\omega}}, w_{0}(x)=e^{-|x|^{2} / 4}$ and $w_{k+1}(x)=\sqrt{2}\left(\frac{d}{d x}+\frac{x}{2}\right) w_{k}$ for all $k \in \mathbb{N}$, that is $\tilde{h}=\sum_{k \in \mathbb{N}} g_{k}(t) h_{k}(y)$, it can be proved that $g_{k} \sim e^{-t}$ for any $k \geq 1$. It follows that $\lim \sup _{t \rightarrow+\infty} \int_{\mathbb{R}}|x|^{4} \tilde{h}(t, x) d x<\infty$, which concludes the proof.

Acknowledgments. A.B. and J.D. have been partially supported ECOS-CONYCIT \# C05E09. J.D. wishes to thank the members of the DIM for their hospitality. M.K. has been partially supported by: FONDECYT 1050311, Nucleo Milenio P04-069-F, FONDAP and ECOS-CONYCIT \# C05E05. A.B. acknowledges the support of the KAUST investigator award. All authors thank an anonymous referee for his careful reading and valuable suggestions, and A.-L. Dalibard for pointing them an error.

(C) 2008 by the authors. This paper may be reproduced, in its entirety, for non-commercial purposes.

\section{REFERENCES}

[1] A. Ajdari and J. Prost, Mouvement induit par un potentiel périodique de basse symétrie: dielectrophorese pulse, C. R. Acad. Sci. Paris, Série II, 315 (1992), p. 1653.

[2] G. Allaire, Homogenization and two-scale convergence, SIAM J. Math. Anal., 23 (1992), pp. $1482-1518$.

[3] C. Ané, S. Blachère, D. Chafaï, P. Fougères, i. Gentil, F. Malrieu, C. Roberto, And G. Scheffer, Sur les inégalités de Sobolev logarithmiques, vol. 10 of Panoramas et Synthèses [Panoramas and Syntheses], Société Mathématique de France, Paris, 2000. Preface by D. Bakry and M. Ledoux.

[4] A. Arnold, J.-P. Bartier, And J. Dolbeault, Interpolation between logarithmic Sobolev and Poincaré inequalities, Commun. Math. Sci., 5 (2007), pp. 971-979.

[5] A. Arnold, P. Markowich, and G. Toscani, On large time asymptotics for drift-diffusionPoisson systems, in Proceedings of the Fifth International Workshop on Mathematical Aspects of Fluid and Plasma Dynamics (Maui, HI, 1998), vol. 29, 2000, pp. 571-581.

[6] A. Arnold, P. Markowich, G. Toscani, and A. Unterreiter, On convex Sobolev inequalities and the rate of convergence to equilibrium for Fokker-Planck type equations, Comm. Partial Differential Equations, 26 (2001), pp. 43-100.

[7] R. D. Astumian, Thermodynamics and kinetics of a brownian motor, Science, 276 (1997), pp. 917-922.

[8] J.-P. Bartier and J. Dolbeault, Convex Sobolev inequalities and spectral gap, C. R. Math. Acad. Sci. Paris, 342 (2006), pp. 307-312. 
[9] J.-P. Bartier, J. Dolbeault, R. Illner, and M. Kowalczyk, A qualitative study of linear drift-diffusion equations with time-dependent or degenerate coefficients, Math. Models Methods Appl. Sci., 17 (2007), pp. 327-362.

[10] W. Beckner, A generalized Poincaré inequality for Gaussian measures, Proc. Amer. Math. Soc., 105 (1989), pp. 397-400.

[11] I. Bena, C. VAn Den Broeck, And M. Copelli, Stokes'drift: a rocking ratchet, J. Stat. Phys., 101 (2000), pp. 415-423.

[12] M. BieR, Brownian ratchets in physics and biology, Contemporary Physics, 38 (1 November 1997), pp. 371-379(9).

[13] M. Bien, Reversals of noise induced flow, Physics Letters A, 211 (1996), pp. 12-18.

[14] A. Blanchet, J. Dolbeault, and M. Kowalczyk, Travelling fronts in stochastic Stokes' drifts, Physica A: Statistical Mechanics and its Applications, 387 (2008), pp. 5741-5751.

[15] M. Borromeo and F. Marchesoni, Brownian surfers, Phys. Lett. A, 249 (1998), pp. 199-203.

[16] E. A. Carlen And M. Loss, Extremals of functionals with competing symmetries, J. Funct. Anal., 88 (1990), pp. 437-456.

[17] D. Chafaї, Entropies, convexity, and functional inequalities: on $\Phi$-entropies and $\Phi$-Sobolev inequalities, J. Math. Kyoto Univ., 44 (2004), pp. 325-363.

[18] M. Chipot, S. Hastings, And D. Kinderlehrer, Transport in a molecular motor system, M2AN Math. Model. Numer. Anal., 38 (2004), pp. 1011-1034.

[19] M. Chipot, D. Kinderlehrer, And M. KowalczyK, A variational principle for molecular motors, Meccanica, 38 (2003), pp. 505-518. Dedicated to Piero Villaggio on the occasion of his 70 th birthday.

[20] D. Cioranescu, A. Damlamian, and G. Griso, Periodic unfolding and homogenization, C. R. Math. Acad. Sci. Paris, 335 (2002), pp. 99-104.

[21] C. V. Den Broeck, Stokes' drift: An exact result, Europhysics Letters, 46 (1999), pp. 1-5.

[22] C. R. Doering, W. Horsthemke, and J. Riordan, Nonequilibrium fluctuation-induced transport, Phys. Rev. Lett., 72 (1994), pp. 2984-2987.

[23] J. Dolbeault And G. Karch, Large time behaviour of solutions to nonhomogeneous diffusion equations, Banach Center Publ., 74 (2006), pp. 133-147.

[24] J. Dolbeault, D. Kinderlehrer, and M. KowalczyK, Remarks about the flashing rachet, in Partial differential equations and inverse problems, vol. 362 of Contemp. Math., Amer. Math. Soc., Providence, RI, 2004, pp. 167-175.

[25] T. Goudon And F. Poupaud, Approximation by homogenization and diffusion of kinetic equations, Comm. Partial Differential Equations, 26 (2001), pp. 537-569.

[26] T. Goudon And F. Poupaud, Homogenization of transport equations: weak mean field approximation, SIAM J. Math. Anal., 36 (2004/05), pp. 856-881 (electronic).

[27] P. Hänggi, F. Marchesoni, And F. Nori, Brownian motors, Ann. Phys. (8), 14 (2005), pp. 51-70.

[28] S. Hastings, D. Kinderlehrer, And J. B. MCLeod, Diffusion mediated transport in multiple state systems, SIAM J. Math. Anal., 39 (2007/08), pp. 1208-1230.

[29] R. Holley and D. Stroock, Logarithmic Sobolev inequalities and stochastic Ising models, J. Statist. Phys., 46 (1987), pp. 1159-1194.

[30] K. M. Jansons and G. D. Lythe, Stochastic Stokes' drift, Phys. Rev. Lett., 81 (1998), pp. 3136-3139.

[31] F. JÜlicher, A. Ajdari, And J. Prost, Modeling molecular motors, Rev. Mod. Phys., 69 (1997), pp. 1269-1282.

[32] D. Kinderlehrer and M. Kowalczyk, Diffusion-mediated transport and the flashing ratchet, Arch. Ration. Mech. Anal., 161 (2002), pp. 149-179.

[33] A. B. Kolomeisky and M. E. Fisher, Molecular motors: a theorist's perspective., Annu. Rev. Phys. Chem., 58 (2007), pp. 675-695.

[34] R. Latala and K. Oleszkiewicz, Between Sobolev and Poincaré, in Geometric aspects of functional analysis, vol. 1745 of Lecture Notes in Math., Springer, Berlin, 2000, pp. 147168.

[35] M. O. Magnasco, Forced thermal ratchets, Phys. Rev. Lett., 71 (1993), pp. 1477-1481.

[36] A. J. Majda and P. R. Kramer, Simplified models for turbulent diffusion: theory, numerical modelling, and physical phenomena, Phys. Rep., 314 (1999), pp. 237-574.

[37] B. Perthame, Transport equations in biology, Frontiers in Mathematics, Birkhäuser Verlag, Basel, 2007.

[38] B. Perthame And P. E. Souganidis, Asymmetric potentials and motor effect: a large deviation approach. Arch. Rat. Mech. Anal., to appear.

[39] P. Reimann, Current reversal in a white noise driven flashing ratchet, Physics Reports, 290 (1997), pp. 149-155. 
[40] _ Brownian motors: noisy transport far from equilibrium, Phys. Rep., 361 (2002), pp. 57265.

[41] P. Reimann and P. HÄngGi, Introduction to the physics of brownian motors, Applied Physics A: Materials Science \& Processing, 75 (2002), pp. 169-178.

[42] O. S. Rотнаus, Logarithmic Sobolev inequalities and the spectrum of Schrödinger operators, J. Funct. Anal., 42 (1981), pp. 110-120.

[43] G. Toscani, Sur l'inégalité logarithmique de Sobolev, C. R. Acad. Sci. Paris Sér. I Math., 324 (1997), pp. 689-694.

[44] A. Unterreiter, A. Arnold, P. Markowich, and G. Toscani, On generalized CsiszárKullback inequalities, Monatsh. Math., 131 (2000), pp. 235-253.

[45] M. Vergassola and M. Avellaneda, Scalar transport in compressible flow, Phys. D, 106 (1997), pp. 148-166. 